\title{
Theory of electron-impact ionization of atoms
}

\author{
A. S. Kadyrov, ${ }^{1, *}$ A. M. Mukhamedzhanov, ${ }^{2}$ A. T. Stelbovics, ${ }^{1}$ and I. Bray ${ }^{1}$ \\ ${ }^{1}$ Centre for Atomic, Molecular and Surface Physics, Division of Science and Engineering, Murdoch University, Perth 6150, Australia \\ ${ }^{2}$ Cyclotron Institute, Texas A\&M University, College Station, Texas 77843, USA
}

(Received 22 April 2004; published 2 December 2004)

\begin{abstract}
The existing formulations of electron-impact ionization of a hydrogenic target suffer from a number of formal problems including an ambiguous and phase-divergent definition of the ionization amplitude. An alternative formulation of the theory is given. An integral representation for the ionization amplitude which is free of ambiguity and divergence problems is derived and is shown to have four alternative, but equivalent, forms well suited for practical calculations. The extension to amplitudes of all possible scattering processes taking place in an arbitrary three-body system follows. A well-defined conventional post form of the breakup amplitude valid for arbitrary potentials including the long-range Coulomb interaction is given. Practical approaches are based on partial-wave expansions, so the formulation is also recast in terms of partial waves and partialwave expansions of the asymptotic wave functions are presented. In particular, expansions of the asymptotic forms of the total scattering wave function, developed from both the initial and the final state, for electronimpact ionization of hydrogen are given. Finally, the utility of the present formulation is demonstrated on some well-known model problems.
\end{abstract}

DOI: $10.1103 /$ PhysRevA.70.062703

PACS number(s): 34.10.+x, 03.65.Nk, 34.80.Dp

\section{INTRODUCTION}

Electron-impact ionization of hydrogenic targets is the simplest three-body Coulomb problem, and, therefore, of fundamental importance. In particular over the last decade the study of this process through direct numerical solution of the relevant Schrödinger equation has emerged as a powerful approach to the problem. The direct method comprising the solution of a set of coupled partial differential equations in a two-dimensional radial lattice was first applied by Temkin [1] to a simplified model of the electron-hydrogen scattering problem which retained only states with zero orbital angular momenta. Later, Poet [2] suggested an efficient numerical approach to this model problem which essentially made it exactly solvable below the first excitation threshold of hydrogen. This model has since been referred to as the TemkinPoet (TP) model and has served as a testing ground for other theoretical approaches. Subsequently, numerical methods were extended to include more partial waves and applied to the full problem at energies between the first and the second excitation thresholds [3-6]. More recently, Jones and Stelbovics [7] used a direct integration method to calculate electron-impact ionization within the framework of the TP model. A major advance in the direct solution of the Schrödinger equation was made using the exterior complex scaling (ECS) technique [8-10] when calculations reached the stage of yielding quantitative agreement with measurements of $e-\mathrm{H}$ fully differential ionization cross sections. Close-coupling-based methods, such as the convergent closecoupling [11], $R$ matrix [12], $T$ matrix [13], and other methods, have also yielded excellent agreement with experiment.

However, from the theory point of view several issues relating to a complete formal understanding of the process

\footnotetext{
*Electronic address: A.Kadyrov@murdoch.edu.au
}

remain open. Considerable progress in numerical computations based on the Scrödinger equation mentioned above has been made in spite of such formal problems. To be more specific, we emphasize, for example, that all of the sophisticated approaches to the ionization problem mentioned above rely on some form of approximation when it comes to extracting the ionization amplitude from the calculated total scattering wave function. In fact, the ionization cross sections are calculated from a formally incomplete definition of the ionization amplitude as we pointed out [14]. Thus, despite the success of the computational methods, the formal theory of ionization has not been able to show how to calculate the ionization amplitude unambiguously. One reason preventing the direct integration methods from extracting ionization cross sections rigorously has been a lack of an ambiguity-free form of the asymptotic wave function for positive energies. The well-known Peterkop asymptotic wave function [15] is not valid in all asymptotic domains relevant to the problem and is ambiguous where it is valid [16]. In part because of this it has been impossible to define the ionization amplitude in a divergence-free manner. The full and unambiguous asymptotic forms of the three-body scattered wave function has been given recently $[16,17]$. This allowed us to obtain an integral representation for the ionization amplitude which is free of ambiguity and divergence problems [14]. In part, our analysis has provided a formal justification of the cross sections obtained in the ECS-based method [8-10].

In this work we give details of the results outlined in [14]. In addition, we present four alternative forms of the ionization amplitude. These forms designed for ionization are then generalized to all possible scattering processes which may take place in the system. Our formulation is also shown to resolve another long-standing formal problem, the extension of the conventional post form of the breakup amplitude, valid for short-range potentials, to long-range potentials. We develop a well-defined post form of the breakup amplitude 
valid for arbitrary potentials including the long-range Coulomb interaction. We then generalize the results for the full amplitudes to the individual partial amplitudes as these are extracted in practical calculations and combined to make up the physical ionization amplitude. This naturally requires knowledge of the partial waves of the asymptotic wave functions. The partial-wave expansion of the asymptotic wave function is developed for both the incident- $[18,19]$ and scattered-wave $[16,17]$ asymptotic forms of the three-body wave functions. Though we deal here with the $e-\mathrm{H}$ system, the formalism presented is general. The results given below are readily applicable to extraction of amplitudes in direct calculations of other atomic and molecular breakup processes including the double photoionization of helium [20] and similar problems in nuclear physics. They may also be useful in further developing the effective-charge perturbation approaches [21-23]. The partial-wave forms of the threebody wave functions presented in this work have other uses. For instance, they are capable of immediately reducing the six-dimensional integrals used in the distorted-wave Born approximation $[19,24,25]$ to two-dimensional ones.

The plan of the paper is as follows. In Sec. II we first discuss the formal and consequent practical problems of the existing theory of ionization. In Sec. III we derive a formally correct, unambiguous, and divergence-free representation for the ionization amplitude. In Sec. IV we show that the ionization amplitude in the present formulation directly takes forms also ideally suited for practical calculations. We demonstrate that there are four alternative forms of the ionization amplitude in addition to the two forms known in the literature as the post and prior forms. These forms are then extended beyond the ionization amplitude to cover also amplitudes of all other possible scattering processes. A partialwave analysis of the initial- and final-state three-body wave functions is performed in Sec. V. Then in Sec. VI we extend the results of Sec. III to the partial ionization amplitudes. The utility of the present formulation of the theory of electronimpact ionization will be demonstrated in Sec. VII for wellknown model problems. The so-called screening and collinear models of electron-hydrogen ionization will be considered. In Secs. VIII and IX we discuss and summarize the results of the present work.

Atomic units are used throughout this work; we also assume that the proton is infinitely heavy compared to the electrons and remains at the origin of the coordinate system. To avoid inessential complications we assume the electrons to be distinguishable. The generalization to the case of indistinguishable electrons (and to arbitrary masses as well) is straightforward.

\section{THE PETERKOP FORMALISM}

Consider scattering of electron $e_{1}$ with incident momentum $\boldsymbol{k}_{i}$ off a hydrogen atom $\left(p, e_{2}\right)$ in initial state $\phi_{i}\left(\boldsymbol{r}_{2}\right)$ of energy $E_{i}$. Assume that the energy of the projectile $k_{i}^{2} / 2$ is enough to break up the target. The ionization amplitude in the prior form is well defined and given according to [26] (for brevity we omit the index prior in the notation; other forms of the amplitude which we introduce later will have a corresponding label)

$$
T\left(\boldsymbol{k}_{1}, \boldsymbol{k}_{2}\right)=\int d \boldsymbol{r}_{1} d \boldsymbol{r}_{2} \Psi_{f}^{-*}\left(\boldsymbol{r}_{1}, \boldsymbol{r}_{2}\right) \bar{V}_{i} \Phi^{(i)}\left(\boldsymbol{r}_{1}, \boldsymbol{r}_{2}\right) .
$$

Here $\Psi_{f}^{-}$is the total scattering wave function developing from an initial state of three particles in the continuum with incoming scattered-wave boundary condition and describes the

$$
e_{1}+e_{2}+p \rightarrow\left\{\begin{array}{l}
e_{1}+e_{2}+p, \\
e_{1}+\left(p, e_{2}\right), \\
e_{2}+\left(p, e_{1}\right)
\end{array}\right.
$$

processes [we call them $3 \rightarrow 3$ scattering and $3 \rightarrow 2$ (recombination) processes]. The wave function $\Psi_{f}^{-}$satisfies the Schrödinger equation

$$
(E-H) \Psi_{f}^{-}\left(\boldsymbol{r}_{1}, \boldsymbol{r}_{2}\right)=0,
$$

where $H=H_{0}+V$ is the total three-body Hamiltonian, $H_{0}=$ $-\Delta_{r_{1}} / 2-\Delta_{r_{2}} / 2$ is the free three-body Hamiltonian, $V$ is the full interaction, and $E=k_{i}^{2} / 2+E_{i}=k_{1}^{2} / 2+k_{2}^{2} / 2$ is the total energy of the system, $\bar{V}_{i}=V-V_{i}$ in Eq. (1) is the interaction of the incident electron with the target particles, $\boldsymbol{r}_{1}$ and $\boldsymbol{r}_{2}$ are the coordinates of the electrons relative to the proton, and $\boldsymbol{k}_{1}$ and $\boldsymbol{k}_{2}$ are their momenta. The wave function representing the initial two-fragment channel is given by a product of the incident plane wave and the initial bound-state wave function

$$
\Phi^{(i)}\left(\boldsymbol{r}_{1}, \boldsymbol{r}_{2}\right)=e^{i k_{i} \cdot \boldsymbol{r}_{1}} \phi_{i}\left(\boldsymbol{r}_{2}\right) .
$$

For further reference we note, however, that, in general, the initial-channel wave function satisfies

$$
\left(E-H_{0}-V_{i}\right) \Phi^{(i)}\left(\boldsymbol{r}_{1}, \boldsymbol{r}_{2}\right)=0,
$$

where $V_{i}$ is the potential responsible for the bound state in the initial channel. According to our particular choice of the initial channel, $V_{i}$ is the Coulomb interaction of electron $e_{2}$ and the proton.

The ionization amplitude given by the form (1) is difficult to calculate because it requires the total scattering wave function $\Psi_{f}^{-}$, which evolves from a free three-particle initial state. In addition, for the ionization amplitude to be calculated from this definition, a knowledge of $\Psi_{f}^{-}$in the entire space is necessary. Therefore, this form of the ionization amplitude has often been used in distorted-wave Born-type calculations (see, e.g., $[19,24,25,27]$ and references therein).

Instead, Peterkop [28,29] and Rudge [30] considered the integral

$$
I_{z_{1}, z_{2}}\left(\boldsymbol{k}_{1}, \boldsymbol{k}_{2}\right)=\int d \boldsymbol{r}_{1} d \boldsymbol{r}_{2} \Phi_{i}^{+}\left(\boldsymbol{r}_{1}, \boldsymbol{r}_{2}\right)(H-E) \Psi_{z_{1}, z_{2}}^{(2 \mathrm{C})-*}\left(\boldsymbol{r}_{1}, \boldsymbol{r}_{2}\right),
$$

where $\Phi_{i}^{+}$is a solution of the Schrödinger equation

$$
(E-H) \Phi_{i}^{+}\left(\boldsymbol{r}_{1}, \boldsymbol{r}_{2}\right)=0,
$$

with outgoing scattered-wave boundary condition [31]. The wave function $\Phi_{i}^{+}$describes 


$$
e_{1}+\left(p, e_{2}\right) \rightarrow\left\{\begin{array}{l}
e_{1}+e_{2}+p, \\
e_{1}+\left(p, e_{2}\right), \\
e_{2}+\left(p, e_{1}\right)
\end{array}\right.
$$

processes [we call them $2 \rightarrow 3$ (ionization) and $2 \rightarrow 2$ scattering processes]. The function $\Psi_{z_{1}, z_{2}}^{(2)-}$ is a product of the two Coulomb (2C) wave functions with effective charges $z_{1}$ and $z_{2}$ :

$$
\Psi_{z_{1}, z_{2}}^{(2 \mathrm{C})-}\left(\boldsymbol{r}_{1}, \boldsymbol{r}_{2}\right)=e^{i \boldsymbol{k}_{1} \cdot \boldsymbol{r}_{1}+i \boldsymbol{k}_{2} \cdot \boldsymbol{r}_{2}} \psi_{z_{1}}\left(\boldsymbol{k}_{1}, \boldsymbol{r}_{1}\right) \psi_{z_{2}}\left(\boldsymbol{k}_{2}, \boldsymbol{r}_{2}\right),
$$

with incoming-wave boundary condition. The Coulomb part is given by

$$
\psi_{\nu}(\boldsymbol{k}, \boldsymbol{r})=\Gamma(1+i \nu / k) \exp (\pi \nu / 2 k){ }_{1} F_{1}(-i \nu / k, 1 ;-i(k r+\boldsymbol{k} \cdot \boldsymbol{r})),
$$

where ${ }_{1} F_{1}$ is the confluent hypergeometric function.

Using Eq. (7) and the Green's theorem [32] the volume integral in Eq. (6) can be written as a surface integral

$$
\begin{aligned}
I_{z_{1}, z_{2}}\left(\boldsymbol{k}_{1}, \boldsymbol{k}_{2}\right)= & \frac{1}{2} \lim _{R \rightarrow \infty} R^{5} \int d \hat{\boldsymbol{r}}_{1} d \hat{\boldsymbol{r}}_{2} \int_{0}^{\pi / 2} d \alpha \sin ^{2} \alpha \\
& \times \cos ^{2} \alpha\left(\Psi_{z_{1}, z_{2}}^{(2 \mathrm{C}) *} \frac{\partial \Phi_{i}^{+}}{\partial R}-\Phi_{i}^{+} \frac{\partial \Psi_{z_{1}, z_{2}}^{(2 \mathrm{C}) *}}{\partial R}\right),
\end{aligned}
$$

where $R=\left(r_{1}^{2}+r_{2}^{2}\right)^{1 / 2}$ is a hyperradius, $\left(\hat{\boldsymbol{r}}_{1}, \hat{\boldsymbol{r}}_{2}, \alpha\right)$ is a fivedimensional hyperangle, with $\alpha=\arctan \left(r_{2} / r_{1}\right)$.

The advantage of the integral form (11) is that here the total wave function $\Phi_{i}^{+}$develops from the exact initial state $\Phi^{(i)}$ given by the product of a plane wave and a hydrogen bound-state wave function. Additionally, Eq. (11) is readily expanded in partial waves leading to a sum of onedimensional integrals. On the other hand Eq. (1) reduces to a two-dimensional integral upon partial-wave expansion. Most importantly, the integral $I_{z_{1}, z_{2}}$ depends only on the asymptotic behavior of the wave functions $\Phi_{i}^{+}$and $\Psi_{z_{1}, z_{2}}^{(2 \mathrm{C})}$ on an infinitely large hypersphere and, therefore, knowledge of the wave function $\Phi_{i}^{+}$over the entire space is not required.

Let us define the domain $\Omega_{0}$ to correspond to the space where all interparticle distances are large, i.e., $r_{1}, r_{2}, r_{3}\left(r_{3}\right.$ $\left.=\boldsymbol{r}_{1}-\boldsymbol{r}_{2}\right) \rightarrow \infty$, in a manner that $r_{1} / r_{2} \rightarrow$ const $\neq 0$. In this domain the asymptotic behavior of $\Phi_{i}^{+}$was found by Peterkop [15] and is written, in the leading order of $R$, as

$$
\Phi_{i}^{+}\left(\boldsymbol{r}_{1}, \boldsymbol{r}_{2}\right) \stackrel{\Omega_{0}}{\rightarrow} A\left(\hat{\boldsymbol{r}}_{1}, \hat{\boldsymbol{r}}_{2}, \alpha\right) R^{-5 / 2} e^{i \kappa R+i \gamma \ln (\kappa R)},
$$

where $\kappa=(2 E)^{1 / 2}$,

$$
\gamma=\frac{1}{\kappa}\left(\frac{1}{\cos \alpha}+\frac{1}{\sin \alpha}-\frac{1}{\sqrt{1-\hat{\boldsymbol{r}}_{1} \cdot \hat{\boldsymbol{r}}_{2} \sin 2 \alpha}}\right),
$$

and $A$ is Peterkop's ionization amplitude. Peterkop showed that the integral $I_{z_{1}, z_{2}}$ exists and differs from amplitude $A$ only by a phase factor:

$$
A\left(\hat{\boldsymbol{k}}_{1}, \hat{\boldsymbol{k}}_{2}, \alpha^{\prime}\right)=\frac{\boldsymbol{\kappa}^{3 / 2}}{(2 \pi)^{5 / 2}} e^{i \beta(R)+i \pi / 4} I_{z_{1}, z_{2}}\left(\boldsymbol{k}_{1}, \boldsymbol{k}_{2}\right),
$$

where $\alpha^{\prime}=\arctan \left(k_{2} / k_{1}\right)$. However, the phase factor $\beta(R)$ diverges as $R \rightarrow \infty$ unless the so-called Peterkop condition

$$
\frac{z_{1}}{k_{1}}+\frac{z_{2}}{k_{2}}=\frac{1}{k_{1}}+\frac{1}{k_{2}}-\frac{1}{\left|\boldsymbol{k}_{1}-\boldsymbol{k}_{2}\right|}
$$

is satisfied. In this case $\beta(R)$ vanishes for large $R$. The relation (14) is known as the Peterkop integral representation for the ionization amplitude.

Thus, in Peterkop's effective-charge approach $z_{1}$ and $z_{2}$ depend on vectors $\boldsymbol{k}_{1}$ and $\boldsymbol{k}_{2}$. For this reason this method was not very useful in practice for it was not clear how to implement condition (15) in realistic calculations. The problems associated in numerical work with the effective-charge approach were discussed recently by McCurdy et al. [33]. Their calculations showed that use of effective charges $z_{1}$ and $z_{2}$ leads to severe numerical problems due to nonorthogonality of the Coulomb wave of a nonunit effective charge to the bound states of hydrogen. From a formal point of view, even if the Peterkop condition were satisfied, one could not establish the ionization amplitude in full. This is because, as mentioned by Peterkop [29], an arbitrary part of the complex amplitude $A$ in asymptotic form (12) can be moved to the phase factor and the resulting wave function would still be a solution to the original Eq. (7) transformed into the six-dimensional hyperspace. Thus, the remaining part of $A$ can equally well be called an ionization amplitude and there is no way of choosing between the different phase possibilities, which is clearly unsatisfactory.

Other formal problems with the scattering theory will be highlighted later. To explain the origin of the problems, we consider the Peterkop formulation summarized by Eqs. (11) and (14), and show that it is incomplete. For further discussion we need to distinguish all possible geometries where the condition $R \rightarrow \infty$ is satisfied. In addition to the $\Omega_{0}$ domain defined earlier, we identify the domain where $r_{1} \rightarrow \infty, r_{2}$ $\rightarrow \infty$ with limited $r_{3}$ as $\Omega_{3}$ and when $r_{2}$ (or $r_{1}$ ) goes to infinity but $r_{1}\left(r_{2}\right)$ remains limited as $\Omega_{2}\left(\Omega_{1}\right)$ :

$$
\begin{gathered}
\Omega_{1}: r_{1} \rightarrow \infty, r_{2} / r_{1} \rightarrow 0, \\
\Omega_{2}: r_{2} \rightarrow \infty, r_{1} / r_{2} \rightarrow 0, \\
\Omega_{3}: r_{1}, r_{2} \rightarrow \infty, r_{3} / r_{1}, r_{3} / r_{2} \rightarrow 0 .
\end{gathered}
$$

For brevity of notation, when $r_{1}$ and $r_{2}$ belong to $\Omega_{i}$ we write this as $R \in \Omega_{i}$. The domains $\Omega_{1}, \Omega_{2}$, and $\Omega_{3}$ correspond to $\alpha \rightarrow \pi / 2, \alpha \rightarrow 0$, and $\alpha \rightarrow \pi / 4$ in the surface integral (11), respectively.

The problems with Peterkop's integral representation for the ionization amplitude originate from the fact that the Peterkop asymptotic form used to calculate the integral (11) is valid only in $\Omega_{0}$. It is clearly seen from Eq. (13) that the Peterkop form cannot be used when $\alpha \rightarrow 0$ and $\alpha \rightarrow \pi / 2$. This wave function is singular also when $\alpha \rightarrow \pi / 4$ if $\hat{\boldsymbol{r}}_{1} \cdot \hat{\boldsymbol{r}}_{2}$ $=1$. At the same time integration over $\alpha$ runs through all these points. Thus, in the integral representation suggested 
by Peterkop the contributions from $\Omega_{1}, \Omega_{2}$, and $\Omega_{3}$ domains are either missing, or taken into account incorrectly. In the next section we show how to generalize the Peterkop formulation to all domains $\Omega_{i}, i=0-3$, of coordinate space.

\section{INTEGRAL REPRESENTATION FOR THE IONIZATION AMPLITUDE}

We begin by noting that the full ambiguity-free asymptotic form of the total scattering wave function $\Phi^{+}$ valid in all asymptotic domains as $R \rightarrow \infty$ has been given recently $[16,17]$. In the present paper we ignore the threebody correlation effects. In electron-impact ionization, due to the unit charge of the electrons and the proton, they contribute in the next-to-the-leading order, and are therefore negligible. However, the importance of these effects may increase with the charge of the particles. Reference [14] shows, in particular, that in the general formulation of the theory for arbitrary particles, three-body correlation effects can easily be incorporated. Without these correlations the full asymptotic wave function may be expressed in the form

$$
\Phi_{i}^{+}\left(\boldsymbol{r}_{1}, \boldsymbol{r}_{2}\right) \stackrel{R \rightarrow \infty}{\sim} \Phi_{1}^{+}\left(\boldsymbol{r}_{1}, \boldsymbol{r}_{2}\right)+\Phi_{2}^{+}\left(\boldsymbol{r}_{1}, \boldsymbol{r}_{2}\right)+\Phi_{c}^{+}\left(\boldsymbol{r}_{1}, \boldsymbol{r}_{2}\right),
$$

where

$$
\begin{gathered}
\Phi_{1}^{+}\left(\boldsymbol{r}_{1}, \boldsymbol{r}_{2}\right)=\Phi^{(i)}\left(\boldsymbol{r}_{1}, \boldsymbol{r}_{2}\right)-\frac{1}{2 \pi} \sum_{n} F\left(k_{n} \hat{\boldsymbol{r}}_{1}, \boldsymbol{k}_{i}\right) \frac{e^{i k_{n} r_{1}}}{r_{1}} \phi_{n}\left(\boldsymbol{r}_{2}\right), \\
\Phi_{2}^{+}\left(\boldsymbol{r}_{1}, \boldsymbol{r}_{2}\right)=-\frac{1}{2 \pi} \sum_{m} G\left(k_{m} \hat{\boldsymbol{r}}_{2}, \boldsymbol{k}_{i}\right) \frac{e^{i k_{m} r_{2}}}{r_{2}} \phi_{m}\left(\boldsymbol{r}_{1}\right),
\end{gathered}
$$

with the summations restricted to discrete target states and

$$
\begin{aligned}
\Phi_{c}^{+}\left(\boldsymbol{r}_{1}, \boldsymbol{r}_{2}\right)= & \frac{1}{(2 \pi)^{5 / 2}} T\left(\frac{\kappa}{R} \boldsymbol{r}_{1}, \frac{\kappa}{R} \boldsymbol{r}_{2}\right) \frac{\kappa^{3 / 2}}{R^{5 / 2}} e^{i \kappa R+i \pi / 4} \\
& \times \psi_{1}\left(\frac{\kappa}{R} \boldsymbol{r}_{1}, \boldsymbol{r}_{1}\right) \psi_{1}\left(\frac{\kappa}{R} \boldsymbol{r}_{2}, \boldsymbol{r}_{2}\right) \psi_{-1 / 2}\left(\frac{\kappa}{2 R} \boldsymbol{r}_{3}, \boldsymbol{r}_{3}\right) .
\end{aligned}
$$

The last factor in Eq. (22) corresponds to the Coulomb interactions between the two electrons. It is defined by Eq. (10) with index $\nu$ referring to the charge-mass factor of the interacting particles. In this case $\nu=-1 / 2$. The ionization amplitude $T$ is as defined according to Eq. (1) (see $[16,17]$ ) and therefore unambiguous. We emphasize that $\Phi_{c}^{+}$, the continuum part of the asymptotic wave function, is valid in all asymptotic domains specified above. In Eqs. (20) and (21) $F$ and $G$ are amplitudes describing the direct and rearrangement scattering. The relative momenta in two-fragment channels are given by $k_{n}=\left[2\left(E-E_{n}\right)\right]^{1 / 2}$.

As our starting point we return to Peterkop's integral form $I_{z_{1}, z_{2}}\left(\boldsymbol{k}_{1}, \boldsymbol{k}_{2}\right)$ defined by Eq. (6). We note that for any large but finite $R$ Peterkop's integral is $R$ dependent even in the case when $z_{1}$ and $z_{2}$ satisfy the Peterkop condition (15). We make this dependence explicit by the notation $I_{z_{1}, z_{2}}\left(\boldsymbol{k}_{1}, \boldsymbol{k}_{2} ; R\right)$. Then, only if $z_{1}$ and $z_{2}$ satisfy the Peterkop condition does the following limit exist:

$$
I_{z_{1}, z_{2}}\left(\boldsymbol{k}_{1}, \boldsymbol{k}_{2}\right)=\lim _{R \rightarrow \infty} I_{z_{1}, z_{2}}\left(\boldsymbol{k}_{1}, \boldsymbol{k}_{2} ; R\right) .
$$

Therefore, in the general case, extra care must be exercised when dealing with such integrals. In this section we first work with finite, but sufficiently large $R$, so that the asymptotic forms (19)-(22) are valid (we call this "asymptotically large $R$ "), until we reach a point where taking the $R \rightarrow \infty$ limit is no longer problematic.

We also note that we can partition the full scattering function $\Phi_{i}^{+}$into a sum of three components whose form is left unspecified in the internal region of the $\left(\boldsymbol{r}_{1}, \boldsymbol{r}_{2}\right)$ space but has the asymptotic behavior (20)-(22). Then the volume integral (6) can be replaced by a sum of three volume integrals, each containing the mentioned components. Now in an analogous way to the standard Peterkop approach we convert each of the integrals into surface integrals for asymptotically large $R$. The full surface integral form then reads

$$
\begin{aligned}
I_{z_{1}, z_{2}}\left(\boldsymbol{k}_{1}, \boldsymbol{k}_{2} ; R\right)= & I_{z_{1}, z_{2}}^{(1)}\left(\boldsymbol{k}_{1}, \boldsymbol{k}_{2} ; R\right)+I_{z_{1}, z_{2}}^{(2)}\left(\boldsymbol{k}_{1}, \boldsymbol{k}_{2} ; R\right) \\
& +I_{z_{1}, z_{2}}^{(c)}\left(\boldsymbol{k}_{1}, \boldsymbol{k}_{2} ; R\right),
\end{aligned}
$$

with

$$
\begin{aligned}
I_{z_{1}, z_{2}}^{(1)}\left(\boldsymbol{k}_{1}, \boldsymbol{k}_{2} ; R\right)= & \frac{1}{2} R^{2} \int d \hat{\boldsymbol{r}}_{1} d \boldsymbol{r}_{2} \\
& \times\left(\Psi_{z_{1}, z_{2}}^{(2 \mathrm{C}) * *} \frac{\partial \Phi_{1}^{+}}{\partial r_{1}}-\Phi_{1}^{+} \frac{\partial \Psi_{z_{1}, z_{2}}^{(2 \mathrm{C})-*}}{\partial r_{1}}\right)_{r_{1}=R},
\end{aligned}
$$

$$
\begin{aligned}
& I_{z_{1}, z_{2}}^{(2)}\left(\boldsymbol{k}_{1}, \boldsymbol{k}_{2} ; R\right)=\frac{1}{2} R^{2} \int d \boldsymbol{r}_{1} d \hat{\boldsymbol{r}}_{2} \\
& \times\left(\Psi_{z_{1}, z_{2}}^{(2 \mathrm{C})-\frac{\partial \Phi_{2}^{+}}{\partial r_{2}}}-\Phi_{2}^{+} \frac{\partial \Psi_{z_{1}, z_{2}}^{(2 \mathrm{C})-*}}{\partial r_{2}}\right)_{r_{2}=R},
\end{aligned}
$$

$$
\begin{aligned}
I_{z_{1}, z_{2}}^{(c)}\left(\boldsymbol{k}_{1}, \boldsymbol{k}_{2} ; R\right)= & \frac{1}{2} R^{5} \int d \hat{\boldsymbol{r}}_{1} d \hat{\boldsymbol{r}}_{2} \int_{0}^{\pi / 2} d \alpha \sin ^{2} \alpha \\
& \times \cos ^{2} \alpha\left(\Psi_{z_{1}, z_{2}}^{(2 \mathrm{C})-*} \frac{\partial \Phi_{c}^{+}}{\partial R}-\Phi_{c}^{+} \frac{\partial \Psi_{z_{1}, z_{2}}^{(2 \mathrm{C}) *}}{\partial R}\right) .
\end{aligned}
$$

The different surfaces follow as a consequence of the different asymptotic forms (20)-(22). It is not difficult to see that

$$
\begin{aligned}
& I_{z_{1}, z_{2}}^{(1)}\left(\boldsymbol{k}_{1}, \boldsymbol{k}_{2} ; R\right) \propto \int d \boldsymbol{r}_{2} e^{-i \boldsymbol{k}_{2} \cdot \boldsymbol{r}_{2}} \psi_{z_{2}}^{*}\left(\boldsymbol{k}_{2}, \boldsymbol{r}_{2}\right) \phi_{n}\left(\boldsymbol{r}_{2}\right), \\
& I_{z_{1}, z_{2}}^{(2)}\left(\boldsymbol{k}_{1}, \boldsymbol{k}_{2} ; R\right) \propto \int d \boldsymbol{r}_{1} e^{-i \boldsymbol{k}_{1} \cdot \boldsymbol{r}_{1}} \psi_{z_{1}}^{*}\left(\boldsymbol{k}_{1}, \boldsymbol{r}_{1}\right) \phi_{m}\left(\boldsymbol{r}_{1}\right),
\end{aligned}
$$


ensuring that these terms would disappear if we were to choose $z_{1}=z_{2}=1$ in violation of the Peterkop condition (15), which we shall now show not to be necessary.

Consider the third integral. As a result of the differential operators for asymptotically large $R$ we write

$$
\begin{aligned}
I_{z_{1}, z_{2}}^{(c)}\left(\boldsymbol{k}_{1}, \boldsymbol{k}_{2} ; R\right)= & \frac{i}{2} R^{5} \int d \hat{\boldsymbol{r}}_{1} d \hat{\boldsymbol{r}}_{2} \int_{0}^{\pi / 2} d \alpha \sin ^{2} \\
& \times \cos ^{2} \alpha\left(\kappa+\cos \alpha \boldsymbol{k}_{1} \cdot \hat{\boldsymbol{r}}_{1}+\sin \alpha \boldsymbol{k}_{2} \cdot \hat{\boldsymbol{r}}_{2}\right) \\
& \times \Phi_{c}^{+}\left(R \cos \alpha \hat{\boldsymbol{r}}_{1}, R \sin \alpha \hat{\boldsymbol{r}}_{2}\right)
\end{aligned}
$$

$$
\begin{aligned}
& \times \exp \left(-i R \cos \alpha \boldsymbol{k}_{1} \cdot \hat{\boldsymbol{r}}_{1}-i R \sin \alpha \boldsymbol{k}_{2} \cdot \hat{\boldsymbol{r}}_{2}\right) \\
& \times \psi_{z_{1}}^{*}\left(\boldsymbol{k}_{1}, R \cos \alpha \hat{\boldsymbol{r}}_{1}\right) \psi_{z_{2}}^{*}\left(\boldsymbol{k}_{2}, R \sin \alpha \hat{\boldsymbol{r}}_{2}\right) .
\end{aligned}
$$

Then using the asymptotic form of the plane wave (see, e.g., [29])

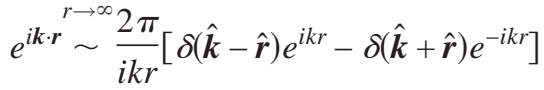

we have, in the leading order,

$$
\begin{aligned}
I_{z_{1}, z_{2}}^{(c)}\left(\boldsymbol{k}_{1}, \boldsymbol{k}_{2} ; R\right)= & \frac{2 \pi^{2}}{i k_{1} k_{2}} R^{3} \int_{0}^{\pi / 2} d \alpha \sin \alpha \cos \alpha\left[\left(\kappa+k_{1} \cos \alpha+k_{2} \sin \alpha\right) \Phi_{c}^{+}\left(R \cos \alpha \hat{\boldsymbol{k}}_{1}, R \sin \alpha \hat{\boldsymbol{k}}_{2}\right) \exp \left(-i R k_{1} \cos \alpha-i R k_{2} \sin \alpha\right)\right. \\
& \times \psi_{z_{1}}^{*}\left(\boldsymbol{k}_{1}, R \cos \alpha \hat{\boldsymbol{k}}_{1}\right) \psi_{z_{2}}^{*}\left(\boldsymbol{k}_{2}, R \sin \alpha \hat{\boldsymbol{k}}_{2}\right)+\left(\kappa-k_{1} \cos \alpha-k_{2} \sin \alpha\right) \Phi_{c}^{+}\left(-R \cos \alpha \hat{\boldsymbol{k}}_{1},-R \sin \alpha \hat{\boldsymbol{k}}_{2}\right) \\
& \times \exp \left(i R k_{1} \cos \alpha+i R k_{2} \sin \alpha\right) \psi_{z_{1}}^{*}\left(\boldsymbol{k}_{1},-R \cos \alpha \hat{\boldsymbol{k}}_{1}\right) \psi_{z_{2}}^{*}\left(\boldsymbol{k}_{2},-R \sin \alpha \hat{\boldsymbol{k}}_{2}\right)-\left(\kappa+k_{1} \cos \alpha-k_{2} \sin \alpha\right) \\
& \times \Phi_{c}^{+}\left(R \cos \alpha \hat{\boldsymbol{k}}_{1},-R \sin \alpha \hat{\boldsymbol{k}}_{2}\right) \exp \left(-i R k_{1} \cos \alpha+i R k_{2} \sin \alpha\right) \psi_{z_{1}}^{*}\left(\boldsymbol{k}_{1}, R \cos \alpha \hat{\boldsymbol{k}}_{1}\right) \psi_{z_{2}}^{*}\left(\boldsymbol{k}_{2},-R \sin \alpha \hat{\boldsymbol{k}}_{2}\right) \\
& -\left(\kappa-k_{1} \cos \alpha+k_{2} \sin \alpha\right) \Phi_{c}^{+}\left(-R \cos \alpha \hat{\boldsymbol{k}}_{1}, R \sin \alpha \hat{\boldsymbol{k}}_{2}\right) \exp \left(i R k_{1} \cos \alpha-i R k_{2} \sin \alpha\right) \psi_{z_{1}}^{*}\left(\boldsymbol{k}_{1},-R \cos \alpha \hat{\boldsymbol{k}}_{1}\right) \\
& \left.\times \psi_{z_{2}}^{*}\left(\boldsymbol{k}_{2}, R \sin \alpha \hat{\boldsymbol{k}}_{2}\right)\right]
\end{aligned}
$$

This is an extremely oscillatory integral and therefore only points of stationary phase in $\alpha$ will contribute in the large- $R$ limit. The first two terms within the square brackets have a common stationary-phase point at $k_{1} \sin \alpha=k_{2} \cos \alpha$, where $\cos \alpha=k_{1} / \kappa$ and $\sin \alpha=k_{2} / \kappa$. Moreover, the second term is identically zero at the stationary point. The third and fourth terms of the integrand have no stationary points and, therefore, do not contribute to the integral. Using Eq. (22) and calculating the remaining integral by means of the stationary-phase method [34] we arrive at

$$
I_{z_{1}, z_{2}}^{(c)}\left(\boldsymbol{k}_{1}, \boldsymbol{k}_{2} ; R\right)=T\left(\boldsymbol{k}_{1}, \boldsymbol{k}_{2}\right) \tau_{z_{1}, z_{2}}\left(\boldsymbol{k}_{1}, \boldsymbol{k}_{2} ; R\right)
$$

where

$$
\begin{aligned}
\tau_{z_{1}, z_{2}}\left(\boldsymbol{k}_{1}, \boldsymbol{k}_{2} ; R\right)= & \psi_{z_{1}}^{*}\left(\boldsymbol{k}_{1}, R \boldsymbol{k}_{1} / \kappa\right) \psi_{z_{2}}^{*}\left(\boldsymbol{k}_{2}, R \boldsymbol{k}_{2} / \kappa\right) \psi_{1}\left(\boldsymbol{k}_{1}, R \boldsymbol{k}_{1} / \boldsymbol{\kappa}\right) \\
& \times \psi_{1}\left(\boldsymbol{k}_{2}, R \boldsymbol{k}_{2} / \kappa\right) \psi_{-1 / 2}\left(\boldsymbol{k}_{3}, 2 R \boldsymbol{k}_{3} / \kappa\right)
\end{aligned}
$$

with $\boldsymbol{k}_{3}=\left(\boldsymbol{k}_{1}-\boldsymbol{k}_{2}\right) / 2$.

Thus the hyperradius-independent physical ionization amplitude factors out. Therefore, one indeed can represent the ionization amplitude in terms of the trial integral (11) but starting from the full specification of the asymptotic form of the scattering wave function through Eqs. (19)-(22) leads to the following form:

$$
\begin{aligned}
& T\left(\boldsymbol{k}_{1}, \boldsymbol{k}_{2}\right) \\
& \quad=\lim _{R \rightarrow \infty} \frac{I_{z_{1}, z_{2}}\left(\boldsymbol{k}_{1}, \boldsymbol{k}_{2} ; R\right)-I_{z_{1}, z_{2}}^{(1)}\left(\boldsymbol{k}_{1}, \boldsymbol{k}_{2} ; R\right)-I_{z_{1}, z_{2}}^{(2)}\left(\boldsymbol{k}_{1}, \boldsymbol{k}_{2} ; R\right)}{\tau_{z_{1}, z_{2}}\left(\boldsymbol{k}_{1}, \boldsymbol{k}_{2} ; R\right)} .
\end{aligned}
$$

Furthermore, $\tau_{z_{1}, z_{2}}$ is well behaved for arbitrary $z_{1}$ and $z_{2}$. Consequently, there is no necessity for $z_{1}$ and $z_{2}$ to satisfy a particular condition in contrast to condition (15) required for the Peterkop amplitude (14). Therefore, one may choose $z_{1}$ and $z_{2}$ such that they maximally simplify the practical calculation of $I_{z_{1}, z_{2}}, I_{z_{1}, z_{2}}^{(1)}$, and $I_{z_{1}, z_{2}}^{(2)}$. The resulting ionization amplitude (35) does not depend on this choice as the $\tau$ factor is adjusted accordingly. Therefore the natural choice is to take $z_{1}=z_{2}=1$. Then, since $I_{1,1}^{(1)}=I_{1,1}^{(2)}=0$ we have

$$
T\left(\boldsymbol{k}_{1}, \boldsymbol{k}_{2}\right)=\lim _{R \rightarrow \infty} \frac{I_{1,1}\left(\boldsymbol{k}_{1}, \boldsymbol{k}_{2} ; R\right)}{\tau_{1,1}\left(\boldsymbol{k}_{1}, \boldsymbol{k}_{2} ; R\right)} .
$$

Note that for practical calculations one would compute $T\left(\boldsymbol{k}_{1}, \boldsymbol{k}_{2} ; R\right) \equiv I_{1,1}\left(\boldsymbol{k}_{1}, \boldsymbol{k}_{2} ; R\right) / \tau_{1,1}\left(\boldsymbol{k}_{1}, \boldsymbol{k}_{2} ; R\right)$ at large $R$ and establish the limit by extrapolation to $R \rightarrow \infty$. This extrapolation procedure is used routinely in the ECS method.

The choice of $z_{1}=z_{2}=1$ was used in the ECS calculations of McCurdy et al. [33]. They experienced serious numerical problems in calculating $I_{z_{1}, z_{2}}$ with the use of effective potentials other than 1 , due to nonorthogonality of the Coulomb 
wave of a nonunit effective charge to bound states of hydrogen. They concluded from their numerical simulations that the optimal choice for $z_{1}$ and $z_{2}$ must be 1 , although such a choice did not follow as a logical consequence of the Peterkop-Rudge formalism they utilized. Our derivation gives a complete theoretical justification of their approach.

Finally, we note some simplifications of Eq. (36) in various kinematic regimes. Provided $k_{1}$ and $k_{2}$ are not too small, which corresponds to the case when $R \in \Omega_{0}$ or $\Omega_{3}$, we can use the asymptotic form of the hypergeometric functions contained in $\psi_{1}$. Then we have

$$
T\left(\boldsymbol{k}_{1}, \boldsymbol{k}_{2}\right)=\lim _{R \rightarrow \infty} \frac{I_{1,1}\left(\boldsymbol{k}_{1}, \boldsymbol{k}_{2} ; R\right)}{\psi_{-1 / 2}\left(\boldsymbol{k}_{3}, 2 R \boldsymbol{k}_{3} / \kappa\right)} .
$$

When neither $k_{1}, k_{2}$, nor $k_{3}$ is too small, which corresponds to the case when $R \in \Omega_{0}$, we have

$$
T\left(\boldsymbol{k}_{1}, \boldsymbol{k}_{2}\right)=\lim _{R \rightarrow \infty} \exp \left[\frac{i}{2 k_{3}} \ln \left(\frac{4 R k_{3}^{2}}{\kappa}\right)\right] I_{1,1}\left(\boldsymbol{k}_{1}, \boldsymbol{k}_{2} ; R\right) .
$$

Now this resembles Peterkop's integral representation (14) but has no additional condition and contains only unambiguously defined quantities. Thus Eq. (38) is the exact integral representation of the physical ionization amplitude in the asymptotic domain where all the interparticle distances are large. This provides proper theoretical justification of the procedure used in direct methods to calculate cross sections, namely,

$$
\left|T\left(\boldsymbol{k}_{1}, \boldsymbol{k}_{2}\right)\right|=\lim _{R \rightarrow \infty}\left|I_{1,1}\left(\boldsymbol{k}_{1}, \boldsymbol{k}_{2} ; R\right)\right| .
$$

Finally, Eqs. (36)-(38) can be used not only to get the correct magnitude of the ionization amplitude but also its ambiguityfree phase part.

\section{ALTERNATIVE FORMS OF THE IONIZATION AND SCATTERING AMPLITUDES}

In the preceding section we showed that the ionization amplitude can be represented in terms of a trial integral which has a structure well suited for practical calculations. However, we can go even further and ask the question: Is it possible to extract the ionization amplitude without recourse to external trial quantities which is the requirement of a formally complete scattering theory? The answer is yes, as we demonstrate in this section.

First we note that Eq. (7) can be written as

$$
(E-H) \Phi_{i}^{(\mathrm{sc})+}\left(\boldsymbol{r}_{1}, \boldsymbol{r}_{2}\right)=\bar{V}_{i} \Phi^{(i)}\left(\boldsymbol{r}_{1}, \boldsymbol{r}_{2}\right),
$$

where we separated the scattered-wave part of $\Phi_{i}^{+}$according to $\Phi_{i}^{(\mathrm{sc})+}=\Phi_{i}^{+}-\Phi^{(i)}$. Combining this with Eq. (3) it follows that

$$
\begin{aligned}
T\left(\boldsymbol{k}_{1}, \boldsymbol{k}_{2}\right) & \equiv\left\langle\Psi_{f}^{-}\left|\bar{V}_{i}\right| \Phi^{(i)}\right\rangle=\left\langle\Psi_{f}^{-}|E-\vec{H}| \Phi_{i}^{(\mathrm{sc})+}\right\rangle \\
& =\left\langle\Psi_{f}^{-}\left|\overleftarrow{H}_{H}-E\right| \Phi_{i}^{(\mathrm{sc})+}\right\rangle+\left\langle\Psi_{f}^{-}|E-\vec{H}| \Phi_{i}^{(\mathrm{sc})+}\right\rangle \\
& =\left\langle\Psi_{f}^{-}\left|\overleftarrow{H}_{0}-\vec{H}_{0}\right| \Phi_{i}^{(\mathrm{sc})+}\right\rangle,
\end{aligned}
$$

where a left (right) arrow on the differential Hamiltonian operator indicates that it acts on the bra (ket) state. This allows us to introduce a new surface-integral form for the ionization amplitude which we denote as $T^{(a)}$ :

$$
\begin{aligned}
T^{(a)}\left(\boldsymbol{k}_{1}, \boldsymbol{k}_{2}\right)= & \frac{1}{2} \lim _{R \rightarrow \infty} R^{5} \int d \hat{\boldsymbol{r}}_{1} d \hat{\boldsymbol{r}}_{2} \int_{0}^{\pi / 2} d \alpha \sin ^{2} \alpha \\
& \times \cos ^{2} \alpha\left(\Psi_{f}^{-*} \frac{\partial \Phi_{i}^{(\mathrm{sc})+}}{\partial R}-\Phi_{i}^{(\mathrm{sc})+} \frac{\partial \Psi_{f}^{-*}}{\partial R}\right) .
\end{aligned}
$$

It is not difficult to verify that due to the asymptotic forms of $\Psi_{f}^{-}$and $\Phi_{i}^{(\mathrm{sc})+}$ no other surface integrals contribute.

Next we show that other forms are also possible. To see this we note that Eq. (3) can be written in the form

$$
\left(E-H_{0}\right) \Psi_{f}^{-}\left(\boldsymbol{r}_{1}, \boldsymbol{r}_{2}\right)=V \Psi_{f}^{-}\left(\boldsymbol{r}_{1}, \boldsymbol{r}_{2}\right) .
$$

In addition, we write Eq. (5) as

$$
\left(E-H_{0}\right) \Phi^{(i)}\left(\boldsymbol{r}_{1}, \boldsymbol{r}_{2}\right)=V_{i} \Phi^{(i)}\left(\boldsymbol{r}_{1}, \boldsymbol{r}_{2}\right) .
$$

Taking into account Eqs. (43) and (44) we get

$$
\begin{aligned}
T\left(\boldsymbol{k}_{1}, \boldsymbol{k}_{2}\right) & \equiv\left\langle\Psi_{f}^{-}\left|V-V_{i}\right| \Phi^{(i)}\right\rangle=\left\langle\Psi_{f}^{-}\left|E-\overleftarrow{H}_{0}-\left(E-\vec{H}_{0}\right)\right| \Phi^{(i)}\right\rangle \\
& =-\left\langle\Psi_{f}^{-}\left|\overleftarrow{H}_{0}-\vec{H}_{0}\right| \Phi^{(i)}\right\rangle .
\end{aligned}
$$

This allows us to introduce a second surface-integral form for the ionization amplitude:

$$
T^{(b)}\left(\boldsymbol{k}_{1}, \boldsymbol{k}_{2}\right)=-\frac{1}{2} \lim _{r_{1} \rightarrow \infty} r_{1}^{2} \int d \hat{\boldsymbol{r}}_{1} d \boldsymbol{r}_{2}\left(\Psi_{f}^{-*} \frac{\partial \Phi^{(i)}}{\partial r_{1}}-\Phi^{(i)} \frac{\partial \Psi_{f}^{-*}}{\partial r_{1}}\right) .
$$

Note that the forms $T^{(a)}$ and $T^{(b)}$ are simply different (but equivalent) ways to represent the ionization amplitude $T$.

Subtracting Eq. (45) from Eq. (41) we can observe that

$$
T^{(a)}\left(\boldsymbol{k}_{1}, \boldsymbol{k}_{2}\right)-T^{(b)}\left(\boldsymbol{k}_{1}, \boldsymbol{k}_{2}\right)=\left\langle\Psi_{f}^{-}\left|\overleftarrow{H}_{0}-\vec{H}_{0}\right| \Phi_{i}^{+}\right\rangle .
$$

Equation (47) serves as a bridge to the post form of the ionization amplitude. In order to see this, let us separate the unscattered and scattered parts of wave function $\Psi_{f}^{-}$according to $\Psi_{f}^{-}=\Psi^{(f)-}+\Psi_{f}^{(\mathrm{sc})-}$. Hence Eq. (47) can also be written as

$$
\begin{aligned}
T^{(a)}\left(\boldsymbol{k}_{1}, \boldsymbol{k}_{2}\right)-T^{(b)}\left(\boldsymbol{k}_{1}, \boldsymbol{k}_{2}\right)= & \left\langle\Psi^{(f)-}\left|\overleftarrow{H}_{0}-\vec{H}_{0}\right| \Phi_{i}^{+}\right\rangle \\
& +\left\langle\Psi_{f}^{(\mathrm{sc})-}\left|\overleftarrow{H}_{0}-\vec{H}_{0}\right| \Phi_{i}^{+}\right\rangle .
\end{aligned}
$$

In other words, the right-hand side of Eq. (48) is presumably the difference between two (different) post forms of the ionization amplitude (this is shown to be true presently). Accordingly, we introduce 


$$
\begin{aligned}
T^{(c)}\left(\boldsymbol{k}_{1}, \boldsymbol{k}_{2}\right)= & \left\langle\Psi^{(f)-}\left|\overleftarrow{H}_{0}-\vec{H}_{0}\right| \Phi_{i}^{+}\right\rangle \\
= & \frac{1}{2} \lim _{R \rightarrow \infty} R^{5} \int d \hat{\boldsymbol{r}}_{1} d \hat{\boldsymbol{r}}_{2} \int_{0}^{\pi / 2} d \alpha \sin ^{2} \alpha \\
& \times \cos ^{2} \alpha\left(\Psi^{(f)-*} \frac{\partial \Phi_{i}^{+}}{\partial R}-\Phi_{i}^{+} \frac{\partial \Psi^{(f)-*}}{\partial R}\right)
\end{aligned}
$$

and

$$
\begin{aligned}
T^{(d)}\left(\boldsymbol{k}_{1}, \boldsymbol{k}_{2}\right)= & -\left\langle\Psi_{f}^{(\mathrm{sc})-}\left|\overleftarrow{H}_{0}-\vec{H}_{0}\right| \Phi_{i}^{+}\right\rangle=-\frac{1}{2} \lim _{r_{1} \rightarrow \infty} r_{1}^{2} \\
& \times \int d \hat{\boldsymbol{r}}_{1} d \boldsymbol{r}_{2}\left(\Psi_{f}^{(\mathrm{sc})-*} \frac{\partial \Phi_{i}^{+}}{\partial r_{1}}-\Phi_{i}^{+} \frac{\partial \Psi_{f}^{(\mathrm{sc})-*}}{\partial r_{1}}\right) .
\end{aligned}
$$

Equations (42), (46), (49), and (50) are convenient for numerical calculations as the result depends only on the asymptotic behavior of the scattered wave functions. The way we obtained $T^{(a)}, T^{(b)}, T^{(c)}$, and $T^{(d)}$, however, may seem to the reader to be based on operator algebra with insufficient theoretical support. Therefore we independently show that Eqs. (42), (46), (49), and (50) are indeed different forms of the ionization amplitude originally defined in Eq. (1). In order to do this, we need the asymptotic forms of both $\Phi_{i}^{+}$and $\Psi_{f}^{-}$.

The asymptotic form of $\Phi_{i}^{+}$is given by Eqs. (19)-(22). At the same time we note that following Ref. [17] we also can derive an analogous form of the wave function $\Psi_{f}^{-}$:

$$
\Psi_{f}^{-}\left(\boldsymbol{r}_{1}, \boldsymbol{r}_{2}\right) \sim \Psi_{1}^{-}\left(\boldsymbol{r}_{1}, \boldsymbol{r}_{2}\right)+\Psi_{2}^{-}\left(\boldsymbol{r}_{1}, \boldsymbol{r}_{2}\right)+\Psi_{c}^{-}\left(\boldsymbol{r}_{1}, \boldsymbol{r}_{2}\right),
$$

with

$$
\begin{gathered}
\Psi_{1}^{-}\left(\boldsymbol{r}_{1}, \boldsymbol{r}_{2}\right)=-\frac{1}{2 \pi} \sum_{n} \tilde{F}\left(k_{n} \hat{\boldsymbol{r}}_{1} ; \boldsymbol{k}_{1}, \boldsymbol{k}_{2}\right) \frac{e^{-i k_{n} r_{1}}}{r_{1}} \phi_{n}\left(\boldsymbol{r}_{2}\right), \\
\Psi_{2}^{-}\left(\boldsymbol{r}_{1}, \boldsymbol{r}_{2}\right)=-\frac{1}{2 \pi} \sum_{m} \widetilde{G}\left(k_{m} \hat{\boldsymbol{r}}_{2} ; \boldsymbol{k}_{1}, \boldsymbol{k}_{2}\right) \frac{e^{-i k_{m} r_{2}}}{r_{2}} \phi_{m}\left(\boldsymbol{r}_{1}\right), \\
\Psi_{c}^{-}\left(\boldsymbol{r}_{1}, \boldsymbol{r}_{2}\right)=\Psi_{\boldsymbol{k}_{1}, \boldsymbol{k}_{2}}^{(f)-}\left(\boldsymbol{r}_{1}, \boldsymbol{r}_{2}\right)+\frac{1}{(2 \pi)^{5 / 2}} \widetilde{T}\left(\frac{\kappa}{R} \boldsymbol{r}_{1}, \frac{\kappa}{R} \boldsymbol{r}_{2}\right) \frac{\kappa^{3 / 2}}{R^{5 / 2}} e^{-i \kappa R+i \pi / 4} \\
\times \psi_{1}\left(\boldsymbol{k}_{1}, \boldsymbol{r}_{1}\right) \psi_{1}\left(\boldsymbol{k}_{2}, \boldsymbol{r}_{2}\right) \psi_{-1 / 2}\left(\boldsymbol{k}_{3}, \boldsymbol{r}_{3}\right),
\end{gathered}
$$

where $\widetilde{F}$ and $\widetilde{G}[35]$ are amplitudes of the $3 \rightarrow 2$ recombination to two-fragment channels $n$ (where electron $e_{2}$ is bound) and $m$ (where electron $e_{1}$ is bound), respectively. In Eq. (54) $\widetilde{T}$ is the amplitude of the $3 \rightarrow 3$ process. The wave function $\Psi^{(f)-}$ which belongs to the continuum part $\Psi_{c}^{-}$is the unscattered part of the asymptotic wave function $\Psi_{f}^{-}$.

The general form of the unscattered asymptotic wave function $\Psi^{(f)-}$ has been derived in [36,37]. This form takes into account the three-body correlation effects. As we mentioned earlier, in electron-impact ionization of hydrogen atom, due to the small charge of the particles, these effects contribute in the second order, and are therefore negligibly small. However, this is not the case when the charges of the particles are high. When the three-body correlations are ne- glected the Alt-Mukhamedzhanov form is equivalent to Garibotti-Miraglia wave function [18] [often called the three Coulomb (3C) wave function]. Thus, in this work, the asymptotic wave function $\Psi^{(f)-}$ is taken in the form

$$
\Psi_{\boldsymbol{k}_{1}, \boldsymbol{k}_{2}}^{(f)-}\left(\boldsymbol{r}_{1}, \boldsymbol{r}_{2}\right)=e^{i \boldsymbol{k}_{1} \cdot \boldsymbol{r}_{1}+i \boldsymbol{k}_{2} \cdot \boldsymbol{r}_{2}} \psi_{1}\left(\boldsymbol{k}_{1}, \boldsymbol{r}_{1}\right) \psi_{1}\left(\boldsymbol{k}_{2}, \boldsymbol{r}_{2}\right) \psi_{-1 / 2}\left(\boldsymbol{k}_{3}, \boldsymbol{r}_{3}\right) .
$$

Consider $T^{(a)}$ of Eq. (42) and $T^{(c)}$ of Eq. (49). Calculating the surface integrals making use of the correct asymptotic behavior for $\Psi_{f}^{-}, \Phi_{i}^{(\mathrm{sc})+}, \Psi^{(f)-}$, and $\Phi_{i}^{+}$, all given above, for both $T^{(a)}$ and $T^{(c)}$ we get, after some algebra,

$$
\begin{aligned}
T^{(a, c)}\left(\boldsymbol{k}_{1}, \boldsymbol{k}_{2}\right)= & T\left(\boldsymbol{k}_{1}, \boldsymbol{k}_{2}\right) \lim _{R \rightarrow \infty}\left|\psi_{1}\left(\boldsymbol{k}_{1}, R \boldsymbol{k}_{1} / \kappa\right)\right|^{2} \\
& \times\left|\psi_{1}\left(\boldsymbol{k}_{2}, R \boldsymbol{k}_{2} / \kappa\right)\right|^{2}\left|\psi_{-1 / 2}\left(\boldsymbol{k}_{3}, 2 R \boldsymbol{k}_{3} / \kappa\right)\right|^{2} .
\end{aligned}
$$

From Eqs. (19)-(22) and (51)-(54) one can easily see that all other terms do not contribute to the result. Taking the remaining limit the Coulomb waves reduce to phase factors so we obtain

$$
T^{(a, c)}\left(\boldsymbol{k}_{1}, \boldsymbol{k}_{2}\right)=T\left(\boldsymbol{k}_{1}, \boldsymbol{k}_{2}\right)
$$

Now we consider $T^{(b)}$ of Eq. (46) and $T^{(d)}$ of Eq. (50). They both can be reduced to

$$
T^{(b, d)}\left(\boldsymbol{k}_{1}, \boldsymbol{k}_{2}\right)=-\frac{1}{2} \lim _{r_{1} \rightarrow \infty} r_{1}^{2} \int d \hat{\boldsymbol{r}}_{1} d \boldsymbol{r}_{2}\left(\Psi_{1}^{-*} \frac{\partial \Phi^{(i)}}{\partial r_{1}}-\Phi^{(i)} \frac{\partial \Psi_{1}^{-*}}{\partial r_{1}}\right) .
$$

Calculating this integral using Eqs. (52) and (4) we get

$$
T^{(b, d)}\left(\boldsymbol{k}_{1}, \boldsymbol{k}_{2}\right)=\widetilde{F} *\left(\boldsymbol{k}_{i} ; \boldsymbol{k}_{1}, \boldsymbol{k}_{2}\right) .
$$

Note that $\widetilde{F}^{*}\left(\boldsymbol{k}_{i} ; \boldsymbol{k}_{1}, \boldsymbol{k}_{2}\right)=T\left(\boldsymbol{k}_{1}, \boldsymbol{k}_{2}\right)$ (see [35]). Thus, as we promised to show, Eqs. (42), (46), (49), and (50) are just alternative surface-integral forms of the exact ionization amplitude in the sense of Eq. (1).

Next we prove that the new forms obtained for the ionization amplitudes can be extended to the amplitudes of all other processes taking place in the collisional system. If in the final channel we have a two-fragment state instead of a three-body state then the total wave function developed from the final state (we denote it $\Phi_{f}^{-}$) will be similar to $\Phi_{i}^{+}$[see Eqs. (19)-(22)]. However, all the scattered parts (twofragment and three-particle ones) of this wave function would have to satisfy the incoming-wave boundary condition. Clearly, the unscattered part $\Phi^{(f)}$ of $\Phi_{f}^{-}$would be given in this case by a product of a plane wave and a bound-state wave function similar to $\Phi^{(i)}$ :

$$
\Phi^{(f)}\left(\boldsymbol{r}_{1}, \boldsymbol{r}_{2}\right)=e^{i \boldsymbol{k}_{f} \boldsymbol{r}_{2}} \phi_{f}\left(\boldsymbol{r}_{1}\right) .
$$

Below we first introduce, in analogy with the four forms of the ionization amplitude, surface-integral forms for the amplitude of the rearrangement scattering, and then verify their validity. Thus 
$G^{(a)}\left(\boldsymbol{k}_{f}, \boldsymbol{k}_{i}\right)=\left\langle\Phi_{f}^{-}\left|\overleftarrow{H}_{0}-\vec{H}_{0}\right| \Phi_{i}^{(\mathrm{sc})+}\right\rangle$

$$
=\frac{1}{2} \lim _{r_{2} \rightarrow \infty} r_{2}^{2} \int d \boldsymbol{r}_{1} d \hat{\boldsymbol{r}}_{2}\left(\Phi_{f}^{-*} \frac{\partial \Phi_{i}^{(\mathrm{sc})+}}{\partial r_{2}}-\Phi_{i}^{(\mathrm{sc})+} \frac{\partial \Phi_{f}^{-*}}{\partial r_{2}}\right),
$$

$$
\begin{aligned}
G^{(b)}\left(\boldsymbol{k}_{f}, \boldsymbol{k}_{i}\right) & =-\left\langle\Phi_{f}^{-}\left|\overleftarrow{H}_{0}-\vec{H}_{0}\right| \Phi^{(i)}\right\rangle \\
& =-\frac{1}{2} \lim _{r_{1} \rightarrow \infty} r_{1}^{2} \int d \hat{\boldsymbol{r}}_{1} d \boldsymbol{r}_{2}\left(\Phi_{f}^{-*} \frac{\partial \Phi^{(i)}}{\partial r_{1}}-\Phi^{(i)} \frac{\partial \Phi_{f}^{-*}}{\partial r_{1}}\right),
\end{aligned}
$$

$$
\begin{aligned}
G^{(c)}\left(\boldsymbol{k}_{f}, \boldsymbol{k}_{i}\right) & =\left\langle\Phi^{(f)}\left|\overleftarrow{H}_{0}-\vec{H}_{0}\right| \Phi_{i}^{+}\right\rangle \\
& =\frac{1}{2} \lim _{r_{2} \rightarrow \infty} r_{2}^{2} \int d \boldsymbol{r}_{1} d \hat{\boldsymbol{r}}_{2}\left(\Phi^{(f) *} \frac{\partial \Phi_{i}^{+}}{\partial r_{2}}-\Phi_{i}^{+} \frac{\partial \Phi^{(f) *}}{\partial r_{2}}\right), \\
G^{(d)}\left(\boldsymbol{k}_{f}, \boldsymbol{k}_{i}\right) & =-\left\langle\Phi_{f}^{(\mathrm{sc})-}\left|\overleftarrow{H}_{0}-\vec{H}_{0}\right| \Phi_{i}^{+}\right\rangle \\
& =-\frac{1}{2} \lim _{r_{1} \rightarrow \infty} r_{1}^{2} \int d \hat{\boldsymbol{r}}_{1} d \boldsymbol{r}_{2}\left(\Phi_{f}^{(\mathrm{sc})-*} \frac{\partial \Phi_{i}^{+}}{\partial r_{1}}-\Phi_{i}^{+} \frac{\partial \Phi_{f}^{(\mathrm{sc})-*}}{\partial r_{1}}\right) .
\end{aligned}
$$

Corresponding forms for the direct scattering amplitude $F$ in the bra-ket notation can be obtained from Eqs. (61), (63), (65), and (67) simply by replacing the label $f$ by $i^{\prime}$. However, their surface-integral forms will be somewhat different, so we write them down explicitly:

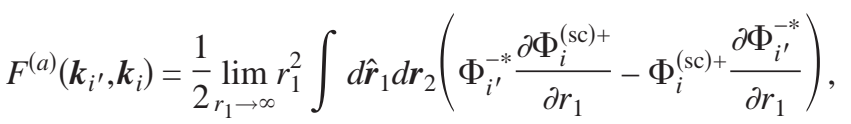

$$
F^{(b)}\left(\boldsymbol{k}_{i^{\prime}}, \boldsymbol{k}_{i}\right)=-\frac{1}{2} \lim _{r_{1} \rightarrow \infty} r_{1}^{2} \int d \hat{\boldsymbol{r}}_{1} d \boldsymbol{r}_{2}\left(\Phi_{i^{\prime}}^{-*} \frac{\partial \Phi^{(i)}}{\partial r_{1}}-\Phi^{(i)} \frac{\partial \Phi_{i^{\prime}}^{-*}}{\partial r_{1}}\right),
$$

$$
F^{(c)}\left(\boldsymbol{k}_{i^{\prime}}, \boldsymbol{k}_{i}\right)=\frac{1}{2} \lim _{r_{1} \rightarrow \infty} r_{1}^{2} \int d \hat{\boldsymbol{r}}_{1} d \boldsymbol{r}_{2}\left(\Phi^{\left(i^{\prime}\right) *} \frac{\partial \Phi_{i}^{+}}{\partial r_{1}}-\Phi_{i}^{+} \frac{\partial \Phi^{\left(i^{\prime}\right) *}}{\partial r_{1}}\right),
$$

$$
\begin{aligned}
F^{(d)}\left(\boldsymbol{k}_{i^{\prime}}, \boldsymbol{k}_{i}\right)= & -\frac{1}{2} \lim _{r_{1} \rightarrow \infty} r_{1}^{2} \int d \hat{\boldsymbol{r}}_{1} d \boldsymbol{r}_{2}\left(\Phi_{i^{\prime}}^{(\mathrm{sc})-*} \frac{\partial \Phi_{i}^{+}}{\partial r_{1}}\right. \\
& \left.-\Phi_{i}^{+} \frac{\partial \Phi_{i^{\prime}}^{(\mathrm{sc})-*}}{\partial r_{1}}\right) .
\end{aligned}
$$

Equations (69)-(72) are also valid for the elastic scattering, when $i^{\prime}=i$ and $k_{i^{\prime}}=k_{i}$.

The new forms for the scattering (both direct and exchange) amplitudes can be verified as follows. Consider, for example, $G^{(a)}$. Using the asymptotic form of the wave func- tions $\Phi_{f}^{-}$and $\Phi_{i}^{(\mathrm{sc})+}$ we can write Eq. (62) in the form

$$
\begin{aligned}
\left\langle\Phi_{f}^{-}\left|\overleftarrow{H}_{0}-\vec{H}_{0}\right| \Phi_{i}^{(\mathrm{sc})+}\right\rangle= & \frac{1}{2} \lim _{r_{2} \rightarrow \infty} r_{2}^{2} \int d \boldsymbol{r}_{1} d \hat{\boldsymbol{r}}_{2}\left(\Phi^{(f) *} \frac{\partial \Phi_{2}^{(\mathrm{sc})+}}{\partial r_{2}}\right. \\
& \left.-\Phi_{2}^{(\mathrm{sc})+} \frac{\partial \Phi^{(f) *}}{\partial r_{2}}\right) .
\end{aligned}
$$

Taking into account Eqs. (21) and (60) and calculating the integrals we have

$$
\left\langle\Phi_{f}^{-}\left|\overleftarrow{H}_{0}-\vec{H}_{0}\right| \Phi_{i}^{(\mathrm{sc})+}\right\rangle=G\left(\boldsymbol{k}_{f}, \boldsymbol{k}_{i}\right) .
$$

Similarly, we can verify the other forms. In addition, note that

$$
\begin{aligned}
G^{(b)}\left(\boldsymbol{k}_{f}, \boldsymbol{k}_{i}\right) & =-\left\langle\Phi_{f}^{-}\left|\overleftarrow{H}_{0}-\vec{H}_{0}\right| \Phi^{(i)}\right\rangle=-\left\langle\Phi_{f}^{-}\left|E-V-\vec{H}_{0}\right| \Phi^{(i)}\right\rangle \\
& =\left\langle\Phi_{f}^{-}\left|\bar{V}_{i}\right| \Phi^{(i)}\right\rangle \equiv G^{(\text {prior })}\left(\boldsymbol{k}_{f}, \boldsymbol{k}_{i}\right)
\end{aligned}
$$

and

$$
\begin{aligned}
G^{(c)}\left(\boldsymbol{k}_{f}, \boldsymbol{k}_{i}\right) & =\left\langle\Phi^{(f)}\left|\overleftarrow{H}_{0}-\vec{H}_{0}\right| \Phi_{i}^{+}\right\rangle=\left\langle\Phi^{(f)}\left|\overleftarrow{H}_{0}+V-E\right| \Phi_{i}^{+}\right\rangle \\
& =\left\langle\Phi^{(f)}\left|\bar{V}_{f}\right| \Phi_{i}^{+}\right\rangle \equiv G^{(\text {post })}\left(\boldsymbol{k}_{f}, \boldsymbol{k}_{i}\right) .
\end{aligned}
$$

In other words, the forms we introduced are easily transformed to the usual prior and post forms of the scattering amplitude.

We also emphasize the importance of the new forms of the ionization amplitude, especially the form $T^{\text {(c) }}$ given by Eq. (49), from the point of view of general scattering theory. Equation (49) leads to a well-defined conventional volumeintegral form of the ionization amplitude in terms of the total three-body scattering wave function $\Phi_{i}^{+}$, being developed from the initial two-fragment channel $\Phi^{(i)}$. In the stationarystate scattering theory the post form of the breakup amplitude is defined by

$$
T^{(\text {post })}\left(\boldsymbol{k}_{1}, \boldsymbol{k}_{2}\right)=\left\langle\boldsymbol{k}_{1}, \boldsymbol{k}_{2}|V| \Phi_{i}^{+}\right\rangle,
$$

where $\left\langle\boldsymbol{r}_{1}, \boldsymbol{r}_{2} \mid \boldsymbol{k}_{1} \boldsymbol{k}_{2}\right\rangle=e^{i \boldsymbol{k}_{1} \cdot \boldsymbol{r}_{1}+i \boldsymbol{k}_{2} \cdot \boldsymbol{r}_{2}}$ is the undistorted three-body plane wave. However, this form is valid only when the interaction between particles is short ranged. The commonly accepted stationary theory of scattering fails to define the same for long-range interactions unless it refers to some screening technique. However, this brings additional problems into play since convergence of the screening procedure when the screening radius is extended to infinity still remains to be proven in the stationary theory of scattering of three charged particles. From the $c$ form of the ionization amplitude we get

$$
\begin{aligned}
T^{(c)}\left(\boldsymbol{k}_{1}, \boldsymbol{k}_{2}\right) & =\left\langle\Psi^{(f)-}\left|\overleftarrow{H}_{0}+V-E-\vec{H}_{0}-V+E\right| \Phi_{i}^{+}\right\rangle \\
& =\left\langle\Psi^{(f)-}\left|\overleftarrow{H}_{0}+V-E\right| \Phi_{i}^{+}\right\rangle .
\end{aligned}
$$

This allows us to introduce

$$
T^{(\text {post })}\left(\boldsymbol{k}_{1}, \boldsymbol{k}_{2}\right)=\left\langle\Psi^{(f)-}|\overleftarrow{H}-E| \Phi_{i}^{+}\right\rangle .
$$

Equation (80) takes the form of Eq. (77) when the full interaction is short ranged. Thus, Eq. (80) extends the definition of the post form of the breakup amplitude to long-range potentials including the Coulomb interaction. 
Finally, we want to make some observations on the implications of our results in this section. First, comparison of the Peterkop integral $I_{1,1}$, obtained from Eq. (11) with $z_{1}=z_{2}=1$, and $T^{(c)}$, given by Eq. (49), reveals that the former is equivalent to extracting the ionization amplitude without the use of the electron-electron correlation. Thus the introduction of the effective charges in the Peterkop formalism is nothing else but an attempt to compensate for the absence of these correlation effects.

Second, in the surface-integral sense the $a$ and $c$ forms of the ionization and scattering amplitudes are identical. The same is true for the $b$ and $d$ forms. This can be seen if we neglect in the appropriate wave functions those parts which do not contribute at all or contribute in higher orders. Thus, the surface-integral forms of Eqs. (42) and (49) can be reduced to (we label this form as $a c$ to distinguish it from the $a$ and $c$ forms)

$$
\begin{aligned}
T^{(a c)}\left(\boldsymbol{k}_{1}, \boldsymbol{k}_{2}\right)= & \frac{1}{2} \lim _{R \rightarrow \infty} R^{5} \int d \hat{\boldsymbol{r}}_{1} d \hat{\boldsymbol{r}}_{2} \int_{0}^{\pi / 2} d \alpha \sin ^{2} \alpha \\
& \times \cos ^{2} \alpha\left(\Psi^{(f)-*} \frac{\partial \Phi_{i}^{(\mathrm{sc})+}}{\partial R}-\Phi_{i}^{(\mathrm{sc})+} \frac{\partial \Psi^{(f)-*}}{\partial R}\right) .
\end{aligned}
$$

Similarly, from Eqs. (46) and (50) we get (we label this as the $b d$ form)

$$
\begin{aligned}
T^{(b d)}\left(\boldsymbol{k}_{1}, \boldsymbol{k}_{2}\right)= & -\frac{1}{2} \lim _{r_{1} \rightarrow \infty} r_{1}^{2} \int d \hat{\boldsymbol{r}}_{1} d \boldsymbol{r}_{2}\left(\Psi_{f}^{(\mathrm{scc})-*} \frac{\partial \Phi^{(i)}}{\partial r_{1}}\right. \\
& \left.-\Phi^{(i)} \frac{\partial \Psi_{f}^{(\mathrm{sc})-*}}{\partial r_{1}}\right) .
\end{aligned}
$$

Accordingly, for the rearrangement scattering amplitudes we obtain

$$
\begin{aligned}
& G^{(a c)}\left(\boldsymbol{k}_{f}, \boldsymbol{k}_{i}\right)=\frac{1}{2} \lim _{r_{2} \rightarrow \infty} r_{2}^{2} \int d \boldsymbol{r}_{1} d \hat{\boldsymbol{r}}_{2}\left(\Phi^{(f) *} \frac{\partial \Phi_{i}^{(\mathrm{sc})+}}{\partial r_{2}}\right. \\
& \left.-\Phi_{i}^{(\mathrm{sc})+} \frac{\partial \Phi^{(f) *}}{\partial r_{2}}\right) \text {, } \\
& G^{(b d)}\left(\boldsymbol{k}_{f}, \boldsymbol{k}_{i}\right)=-\frac{1}{2} \lim _{r_{1} \rightarrow \infty} r_{1}^{2} \int d \hat{\boldsymbol{r}}_{1} d \boldsymbol{r}_{2}\left(\Phi_{f}^{(\mathrm{sc})-*} \frac{\partial \Phi^{(i)}}{\partial r_{1}}\right. \\
& \left.-\Phi^{(i)} \frac{\partial \Phi_{f}^{(\mathrm{sc})-*}}{\partial r_{1}}\right) \text {, }
\end{aligned}
$$

and completely analogously for the direct scattering amplitudes we have

$$
\begin{aligned}
F^{(a c)}\left(\boldsymbol{k}_{i^{\prime}}, \boldsymbol{k}_{i}\right)= & \frac{1}{2} \lim _{r_{1} \rightarrow \infty} r_{1}^{2} \int d \hat{\boldsymbol{r}}_{1} d \boldsymbol{r}_{2}\left(\Phi^{\left(i^{\prime}\right) *} \frac{\partial \Phi_{i}^{(\mathrm{sc})+}}{\partial r_{1}}\right. \\
& \left.-\Phi_{i}^{(\mathrm{sc})+} \frac{\partial \Phi^{\left(i^{\prime}\right) *}}{\partial r_{1}}\right)
\end{aligned}
$$

$$
\begin{aligned}
F^{(b d)}\left(\boldsymbol{k}_{i^{\prime}}, \boldsymbol{k}_{i}\right)= & -\frac{1}{2} \lim _{r_{1} \rightarrow \infty} r_{1}^{2} \int d \hat{\boldsymbol{r}}_{1} d \boldsymbol{r}_{2}\left(\Phi_{i^{\prime}}^{(\mathrm{sc})-*} \frac{\partial \Phi^{(i)}}{\partial r_{1}}\right. \\
& \left.-\Phi^{(i)} \frac{\partial \Phi_{i^{\prime}}^{(\mathrm{sc})-*}}{\partial r_{1}}\right) .
\end{aligned}
$$

These forms are recommended for practical calculations since they have a simpler structure. Which form (either $a c$ or $b d$ ) to use in a particular case depends on which form of the scattered wave function, either $\Phi_{i}^{(\mathrm{sc})+}$ or $\Psi_{f}^{(\mathrm{sc})-}$, is chosen to be calculated numerically. So far, most approaches to the problem are based on calculations of $\Phi_{i}^{+}$. Therefore, to give an illustration of the use of the formalism, assume that we were able to solve the Schrödinger equation and find $\Phi_{i}^{(\mathrm{sc})+}$ at asymptotically large distances. Then the ionization amplitude is extracted using $T^{(a c)}$ [Eq. (81)]. For all that, the asymptotic value of the other participating wave function $\Psi^{(f)-}$ is taken from Eq. (55). The exchange amplitudes are calculated using $G^{(a c)}$ [Eq. (83)]. The asymptotic value of $\Phi^{(f}$ is calculated from Eq. (60). Similarly, the direct scattering amplitudes are calculated using $F^{(a c)}$ [Eq. (85)]. The asymptotic value $\Phi^{(i)}$ is taken from Eq. (4). The remaining $b d$ forms are reserved for extracting the corresponding amplitudes when the Schrödinger equation is solved for the total scattering wave function being developed from the final state.

Third, calculations using the prior form of the ionization amplitude in the Born approximation $T_{\mathrm{Born}}\left(\boldsymbol{k}_{1}, \boldsymbol{k}_{2}\right)$ $=\left\langle\Psi^{(f)}-\left|\bar{V}_{i}\right| \Phi^{(i)}\right\rangle$ became popular using the influential work of Brauner et al. [19]. They helped in our understanding of the dynamics of the electron-impact ionization process at intermediate to high energies. In the light of the usefulness of the prior form of the ionization Born amplitude it would be interesting to see what is the capacity of the Born approximation based on the post form of the amplitude $T_{\text {Born }}^{\text {(post) }}\left(\boldsymbol{k}_{1}, \boldsymbol{k}_{2}\right)$ $=\left\langle\Psi^{(f)-}|\overleftarrow{H}-E| \Phi^{(i)}\right\rangle$ which we obtain from Eq. (80).

In concluding this section we emphasize that the results given here demonstrate the self-consistency of the theory when it is formulated in a correct fashion. Furthermore, in the formalism described above we have not been required to reference the masses of the particles or the explicit forms of the interactions between them. This makes it obvious that the amplitudes of all processes (elastic scattering, direct excitation, rearrangement, and breakup) in an arbitrary three-body system can be directly written in the surface-integral formalism developed here. The fact that we assumed that within the two-fragment channels there is no residual Coulomb interaction is not an essential factor. If there is such an interaction in these channels, one needs to replace the respective plane waves in the asymptotic wave functions with the corresponding Coulomb-modified ones; however, this does not change the results given above or their proof.

\section{THE PARTIAL-WAVE ANALYSIS OF THE INITIAL AND FINAL STATE THREE-BODY WAVE FUNCTIONS}

The process of solving the electron-impact ionization problem is twofold. First, one has to find the total scattering wave function, and, second, to extract from it the necessary 
ionization information. Generally speaking, the direct numerical integration of the Schrödinger equation (7) for electron-hydrogen ionization is carried out for the partialwave form of the equation. In order to derive the equation we require the total three-body wave function $\Phi_{i}^{+}$to be expanded in bipolar spherical harmonics of a pair of unit vectors $\hat{\boldsymbol{r}}_{1}$ and $\hat{\boldsymbol{r}}_{2}$ :

$$
\Phi_{i}^{+}\left(\boldsymbol{r}_{1}, \boldsymbol{r}_{2}\right)=\sum_{l_{1}, l_{2}, L, M} \mathcal{R}_{l_{1}, l_{2}, L, M}^{+}\left(r_{1}, r_{2}\right) \mathcal{Y}_{l_{1}, l_{2}, L, M}\left(\hat{\boldsymbol{r}}_{1}, \hat{\boldsymbol{r}}_{2}\right),
$$

with the bipolar spherical harmonics defined as [38]

$$
\mathcal{Y}_{l_{1}, l_{2}, L, M}\left(\hat{\boldsymbol{r}}_{1}, \hat{\boldsymbol{r}}_{2}\right)=\sum_{m_{1}, m_{2}} C_{l_{1} m_{1} l_{2} m_{2}}^{L M} Y_{l_{1}, m_{1}}\left(\hat{\boldsymbol{r}}_{1}\right) Y_{l_{2}, m_{2}}\left(\hat{\boldsymbol{r}}_{2}\right),
$$

where $C_{l_{1} m_{1} l_{2} m_{2}}^{L M}$ are the Clebsch-Gordan coefficients, $Y_{l_{1}, m_{1}}\left(\hat{\boldsymbol{r}}_{1}\right)$ are the spherical harmonics, $l_{1}$ and $l_{2}$ are the angular momenta of electrons, while $L$ is the total angular momentum and $m_{1}, m_{2}$, and $M$ are the projections of $l_{1}, l_{2}$, and $L$, respectively. Here the triad of angular momenta $\left\{l_{1}, l_{2}, L\right\}$ satisfies the well-known triangular conditions. Below, when we introduce more angular momenta, similar conditions are implicitly assumed for appropriate triads. The radial coefficients are defined as

$$
\mathcal{R}_{l_{1}, l_{2}, L, M}^{+}\left(r_{1}, r_{2}\right)=\int d \hat{\boldsymbol{r}}_{1} d \hat{\boldsymbol{r}}_{2} \Phi_{i}^{+}\left(\boldsymbol{r}_{1}, \boldsymbol{r}_{2}\right) \mathcal{Y}_{l_{1}, l_{2}, L, M}^{*}\left(\hat{\boldsymbol{r}}_{1}, \hat{\boldsymbol{r}}_{2}\right)
$$

Expansion (87) transforms Eq. (7) into an infinite set of twodimensional second-order partial differential equations for radial waves $\mathcal{R}_{l_{1}, l_{2}, L, M}^{+}\left(r_{1}, r_{2}\right)$. Then the infinite set is truncated and solved in a two-dimensional $\left(r_{1}, r_{2}\right)$ lattice, e.g., using standard numerical techniques like finite-element or finite-difference methods, imposing proper boundary conditions. However, in this work we will not discuss the ways of solving the aforementioned set of equations. Rather, we assume that we are able to obtain reliable numerical solutions for the radial waves $\mathcal{R}_{l_{1}, l_{2}, L, M}^{+}\left(r_{1}, r_{2}\right)$. Here we are interested in the second phase of the solution process; namely, we will answer the question of how to extract the partial ionization amplitudes from the wave functions $\mathcal{R}_{l_{1}, l_{2}, L, M}^{+}\left(r_{1}, r_{2}\right)$. This requires a knowledge of the analytic form of the partial waves in the asymptotic domains relevant to ionization. In this section we will derive the asymptotic form of the expansion coefficients $\mathcal{R}_{l_{1}, l_{2}, L, M}^{+}\left(r_{1}, r_{2}\right)$. Further, we will generalize the results of Sec. III given for the full physical ionization amplitude to individual partial ionization amplitudes and establish an integral representation for them.

The partial-wave expansion of the asymptotic three-body wave function is a long-standing problem. In the first attempt made by Peterkop and Rabik [39] a Fourier-like expansion was used. Later Altick [40] found the monopole term of the partial asymptotic wave function using a multipoleexpansion approach, where the electron-electron interaction potential is first expanded in terms of the Legendre polynomials. In subsequent publications $[41,42]$ he showed how to extend the monopole-term solution to the dipole term. Later Peterkop and Liepinsh [43-45] and Peterkop and Gailitis [46] also tried to utilize a somewhat similar technique. In the end, neither Altick, nor Peterkop and co-workers could go beyond the $L=0$ partial wave, although they argued that their procedures in principle could be generalized to the higher partial waves. Even for the $L=0$ partial wave, the aforementioned works were not able to provide clear-cut, practically useful wave functions for the simplest cases, like the Temkin-Poet or collinear $S$-wave models. For instance, Altick [41,42] suggested that the centrifugal forces corresponding to the orbital motion of the electrons do not contribute and neglected them. This is equivalent to setting $l_{1}$ $=l_{2}=0$. This fact alone is enough to understand that his results are approximate even for the $L=0$ partial wave where other $l_{1}=l_{2} \neq 0$ also contribute. We shall demonstrate that his results are not exact even for the Temkin-Poet model, where all angular momenta are zero. The only exact result was given by Merkuriev and Faddeev [47], but for the $S$-wave asymptotic wave function and only for the case of three identical particles (i.e., repulsive potentials). In atomic physics where attractive potentials are present it is not clear whether the Merkuriev-Faddeev derivation is valid. The reason for this is that, as pointed out by Merkuriev and Faddeev [47], if one of the particles has a charge of the opposite sign then at some points the asymptotic forms of the Faddeev components diverge. On the whole, these attempts to derive the partial-wave expansions have not succeeded.

The difficulty of the situation was encapsulated by Gailitis $[48,49]$, who came to a very general conclusion that a state of any system of three or more free particles with long-range interactions in the final (or initial) state "cannot be described by the angular momenta of the particles." Hence, the partialwave expansion of the total wave function "becomes meaningless." According to Gailitis this was a consequence of the long range of the Coulomb interaction.

The analytic form of Eq. (22) suggests that indeed it is not possible to expand it in partial waves in a straightforward manner. This is due to the dependence of both the ionization amplitude $T$ and the electron-electron correlation term $\psi_{-1 / 2}$ in Eq. (22) on the vectors $\boldsymbol{r}_{1}$ and $\boldsymbol{r}_{2}$. Any direct expansion of $\Phi_{c}^{+}$as a consequence will lead to a result where each partial wave function depends on an infinite set of all possible partial ionization amplitudes. The same conclusion is applicable to the Peterkop form (12). It is in this formal difficulty that the Gailitis conclusion is founded. These problems do not arise provided the interaction between the particles is short ranged.

However, this is where our agreement with Gailitis ends. One must recognize and proceed from the fact that all known direct integration approaches to the ionization problem assume the possibility of a partial-wave expansion of the threebody wave function. Even though they use one or another approximation where necessary, they solve the problem for individual partial waves. The reasonably fast convergence of the partial-wave summation and the generally good results they give indicate that their implicit assumption of the viability of a partial-wave method is not groundless. With the body 
of computational evidence of the efficacy and convergence of partial-wave expansions providing motivation we show that the partial-wave expansion does make sense. We demonstrate that the asymptotic three-body wave functions can be expanded in partial waves. Moreover, the final results we get are exact in the leading order. They have simple and clear analytical forms especially for the model cases mentioned in the preceding paragraph. In the process of derivation we also identify the reason why the final asymptotic form of the scattered wave was not expandable in partial waves.

Our method of attack is based on using the fundamental asymptotic relationship between the unscattered and scattered three-body wave functions, which has been established recently $[16,17]$. To be specific, we first expand $\Psi^{(f)-}$, then using the relationship between $\Phi_{c}^{+}$and $\Psi^{(f)-}$ calculate partial waves of $\Phi_{c}^{+}$.

\section{A. Asymptotic wave function $\Psi_{k_{1}, k_{2}}^{(f)-}\left(r_{1}, r_{2}\right)$ in partial waves}

Let us separate all the angular information contained in the wave function $\Psi_{\boldsymbol{k}_{1}, \boldsymbol{k}_{2}}^{(f)-}\left(\boldsymbol{r}_{1}, \boldsymbol{r}_{2}\right)$ according to the expansion in a complete set of bispherical harmonics:

$$
\Psi_{\boldsymbol{k}_{1}, \boldsymbol{k}_{2}}^{(f)-}\left(\boldsymbol{r}_{1}, \boldsymbol{r}_{2}\right)=\sum_{\lambda \lambda^{\prime}} \mathcal{R}_{\lambda, \lambda^{\prime}}^{-}\left(k_{1}, k_{2} ; r_{1}, r_{2}\right) \mathcal{Y}_{\lambda^{\prime}}^{*}\left(\hat{\boldsymbol{k}}_{1}, \hat{\boldsymbol{k}}_{2}\right) \mathcal{Y}_{\lambda}\left(\hat{\boldsymbol{r}}_{1}, \hat{\boldsymbol{r}}_{2}\right),
$$

where $\lambda=\left\{l_{1}, l_{2}, L, M\right\}$ and $\lambda^{\prime}=\left\{l_{1}^{\prime}, l_{2}^{\prime}, L^{\prime}, M^{\prime}\right\}$. We are interested in the radial coefficients $\mathcal{R}_{\lambda, \lambda^{\prime}}^{-}\left(r_{1}, r_{2}\right)$, which are defined by

$$
\begin{aligned}
& \mathcal{R}_{\lambda, \lambda^{\prime}}^{-}\left(k_{1}, k_{2} ; r_{1}, r_{2}\right) \\
& \quad=\int d \hat{\boldsymbol{k}}_{1} d \hat{\boldsymbol{k}}_{2} d \hat{\boldsymbol{r}}_{1} d \hat{\boldsymbol{r}}_{2} \Phi_{\boldsymbol{k}_{1}, \boldsymbol{k}_{2}}^{(f)-}\left(\boldsymbol{r}_{1}, \boldsymbol{r}_{2}\right) \mathcal{Y}_{\lambda^{\prime}}\left(\hat{\boldsymbol{k}}_{1}, \hat{\boldsymbol{k}}_{2}\right) \mathcal{Y}_{\lambda}^{*}\left(\hat{\boldsymbol{r}}_{1}, \hat{\boldsymbol{r}}_{2}\right) .
\end{aligned}
$$

To begin the derivation, let us expand the Coulomb wave function $e^{i \boldsymbol{k} \cdot \boldsymbol{r}} \psi_{1}(\boldsymbol{k}, \boldsymbol{r})$ according to

$$
e^{i \boldsymbol{k} \cdot \boldsymbol{r}} \psi_{1}(\boldsymbol{k}, \boldsymbol{r})=\sum_{l, m} i^{l} e^{-i \sigma_{l}(k)} \varphi_{l}(k, r) Y_{l, m}^{*}(\hat{\boldsymbol{k}}) Y_{l, m}(\hat{\boldsymbol{r}}),
$$

where

$$
\sigma_{l}(k)=\arg \Gamma(l+1-i / k)
$$

is the Coulomb phase shift. The radial Coulomb functions $\varphi_{l}(k, r)$ are given by

$$
\begin{aligned}
\varphi_{l}(k, r)= & \frac{4 \pi}{e^{-\pi / 2 k}} \frac{|\Gamma(l+1-i / k)|}{\Gamma(2 l+2)} e^{i k r}(2 k r)^{l} \\
& \times{ }_{1} F_{1}(l+1-i / k ; 2 l+2 ;-2 i k r) .
\end{aligned}
$$

We emphasize that our Coulomb wave function satisfies the incoming-wave boundary condition. The phase factor $e^{-i \sigma_{l}(k)}$ in Eq. (92) reflects this fact. In case of the outgoing-wave boundary condition this factor would change to $e^{i \sigma_{l}(k)}$.

The distortion factor $\psi_{-1 / 2}(\boldsymbol{k}, \boldsymbol{r})$ is expanded as

$$
\psi_{-1 / 2}(\boldsymbol{k}, \boldsymbol{r})=\sum_{l, m} \chi_{l}(k, r) Y_{l, m}^{*}(\hat{\boldsymbol{k}}) Y_{l, m}(\hat{\boldsymbol{r}}) .
$$

For the expansion coefficients $\chi_{l}(k, r)$, after some algebra, we derive

$$
\begin{aligned}
\chi_{l}(k, r)= & \lim _{\epsilon \rightarrow 0} \frac{4 \pi}{e^{\pi / 4 k}} \frac{\Gamma(1-i / 2 k)}{\Gamma(2 l+2)} \frac{\Gamma(\epsilon+l+i / 2 k)}{\Gamma(\epsilon+i / 2 k)}(-2 i k r){ }_{1}^{l} F_{1}(\epsilon \\
& +l+i / 2 k ; 2 l+2 ;-2 i k r) .
\end{aligned}
$$

Note that the limiting procedure in the above equation has been introduced simply to show that the expansion also holds for the case when there is no electron-electron interaction. In this case setting the Coulomb charge to 0 , due to the $\Gamma(\epsilon$ $+l) / \Gamma(\epsilon)$ factor, we have $\chi_{l}(k, r)=4 \pi \delta_{l 0}$. Having noted this we implicitly assume the limiting procedure in the following and simply set $\epsilon=0$ in Eq. (96).

Then we have

$$
\begin{aligned}
\Psi_{\boldsymbol{k}_{1}, \boldsymbol{k}_{2}}^{(f)-}\left(\boldsymbol{r}_{1}, \boldsymbol{r}_{2}\right)= & \sum_{\substack{l_{3}, l_{4}, l_{5}, m_{3}, m_{4}, m_{5}}} i^{l_{3}+l_{4}} e^{-i \sigma_{l_{3}}\left(k_{1}\right)-i \sigma_{l_{4}}\left(k_{2}\right)} \varphi_{l_{3}}\left(k_{1}, r_{1}\right) \varphi_{l_{4}}\left(k_{2}, r_{2}\right) \\
& \times \chi_{l_{5}}\left(k_{3}, r_{3}\right) Y_{l_{3}, m_{3}}^{*}\left(\hat{\boldsymbol{k}}_{1}\right) Y_{l_{4}, m_{4}}^{*}\left(\hat{\boldsymbol{k}}_{2}\right) Y_{l_{5}, m_{5}}^{*}\left(\hat{\boldsymbol{k}}_{3}\right) \\
& \times Y_{l_{3}, m_{3}}\left(\hat{\boldsymbol{r}}_{1}\right) Y_{l_{4}, m_{4}}\left(\hat{\boldsymbol{r}}_{2}\right) Y_{l_{5}, m_{5}}\left(\hat{\boldsymbol{r}}_{3}\right) .
\end{aligned}
$$

Expanding $Y_{l_{5}, m_{5}}\left(\hat{\boldsymbol{r}}_{3}\right)$ and $Y_{l_{5}, m_{5}}\left(\hat{\boldsymbol{k}}_{3}\right)$ in terms of the spherical harmonics of vectors $\hat{\boldsymbol{r}}_{1}, \hat{\boldsymbol{r}}_{2}$, and $\hat{\boldsymbol{k}}_{1}, \hat{\boldsymbol{k}}_{2}$, respectively, we get

$$
\begin{aligned}
& \Psi_{\boldsymbol{k}_{1}, \boldsymbol{k}_{2}}^{(f)-}\left(\boldsymbol{r}_{1}, \boldsymbol{r}_{2}\right)=\sum_{\substack{l_{3}, l_{4}, l_{5}, m_{3}, m_{4}, m_{5}}} \hat{l}_{3}^{2} \hat{l}_{4}^{2} i^{l_{3}+l_{4}} e^{-i \sigma_{l_{3}}\left(k_{1}\right)-i \sigma_{l_{4}}\left(k_{2}\right)} \varphi_{l_{3}}\left(k_{1}, r_{1}\right) \varphi_{l_{4}}\left(k_{2}, r_{2}\right) \frac{\chi_{l_{5}}\left(k_{3}, r_{3}\right)}{\left(k_{3} r_{3}\right)^{l_{5}}} \frac{\left(2 l_{5}+1\right) !}{4 \pi} \\
& \times \sum_{\substack{l_{6}, l_{8}, m_{6}, m_{8}}} \frac{(-1)^{l_{7}+l_{9}}}{2^{l_{8}+l_{9}}} \frac{r_{1}^{l_{6}} r_{2}^{l_{7}} k_{1}^{l_{8}} k_{2}^{l_{9}}}{\left[\left(2 l_{6}\right) !\left(2 l_{7}\right) !\left(2 l_{8}\right) !\left(2 l_{9}\right) !\right]^{1 / 2}} C_{l_{6} m_{6} m_{6} l_{7} m_{7}} C_{l_{8} m_{8} m_{5} l_{9} m_{9}} \sum_{\substack{l_{10}, l_{11}, l_{12}, l_{13}, m_{10}, m_{11}, m_{12}, m_{13}}} \frac{1}{\hat{l}_{10} \hat{l}_{11} \hat{l}_{12} \hat{l}_{13}} \\
& \times C_{l_{3} m_{3} l_{6} m_{6}}^{l_{10} m_{10}} C_{l_{3} 0 l_{6} 0}^{l_{10} 0} C_{l_{4} m_{4} l_{7} m_{7}}^{l_{11} m_{11}} C_{l_{4} 0 l_{7} 0}^{l_{110} 0} C_{l_{3} m_{3} l_{8} m_{8}}^{l_{12} m_{12}} C_{l_{3} 0 l_{8} 0}^{l_{11} 0} C_{l_{4} m_{4} l_{9} m_{9}}^{l_{13} m_{13}} C_{l_{4} 0 l_{9} 0}^{l_{13} 0} Y_{l_{10}, m_{10}}\left(\hat{\boldsymbol{r}}_{1}\right) Y_{l_{11}, m_{11}}\left(\hat{\boldsymbol{r}}_{2}\right) Y_{l_{12}, m_{12}}^{*}\left(\hat{\boldsymbol{k}}_{1}\right) Y_{l_{13}, m_{13}}^{*}\left(\hat{\boldsymbol{k}}_{2}\right),
\end{aligned}
$$


where $l_{7}=l_{5}-l_{6}$ and $l_{9}=l_{5}-l_{8}$ and $\hat{l}=\sqrt{2 l+1}$.

Now we have to consider

$$
\frac{\chi_{l_{5}}\left(k_{3}, r_{3}\right)}{\left(k_{3} r_{3}\right)^{l_{5}}} \propto{ }_{1} F_{1}\left(l_{5}+i / 2 k_{3} ; 2 l_{5}+2 ;-2 i k_{3} r_{3}\right),
$$

which contains information on the directions of $\boldsymbol{r}_{1}$ and $\boldsymbol{r}_{2}$. We expand the hypergeometric function according to

$$
\begin{aligned}
{ }_{1} F_{1} & \left(l_{5}+i / 2 k_{3} ; 2 l_{5}+2 ;-2 i k_{3} r_{3}\right) \\
& =\sum_{l_{14}, m_{14}} g_{l_{5}, l_{14}}\left(k_{3} ; r_{1}, r_{2}\right) Y_{l_{14}, m_{14}}^{*}\left(\hat{\boldsymbol{r}}_{1}\right) Y_{l_{14}, m_{14}}\left(\hat{\boldsymbol{r}}_{2}\right),
\end{aligned}
$$

with

$$
\begin{aligned}
g_{l_{5}, l_{14}}\left(k_{3} ; r_{1}, r_{2}\right)= & 2 \pi \int_{-1}^{1} d x_{1} F_{1}\left(l_{5}+i / 2 k_{3} ; 2 l_{5}+2 ;-2 i k_{3} r_{3}\right) \\
& \times P_{l_{14}}(x),
\end{aligned}
$$

where $x=\hat{\boldsymbol{r}}_{1} \cdot \hat{\boldsymbol{r}}_{2}$. Then the final expansion, which helps couple all of the introduced orbital angular momenta, is

$$
\begin{aligned}
& (-2 i)^{l_{5}} \frac{\Gamma\left(1-i / 2 k_{3}\right)}{e^{\pi / 4 k_{3}}} \frac{\Gamma\left(l_{5}+i / 2 k_{3}\right)}{\Gamma\left(i / 2 k_{3}\right)} g_{l_{5}, l_{14}}\left(k_{3} ; r_{1}, r_{2}\right) \\
& =\sum_{l_{15}, m_{15}} d_{l_{5}, l_{14}, l_{15}}\left(k_{1}, k_{2} ; r_{1}, r_{2}\right) Y_{l_{15}, m_{15}}^{*}\left(\hat{\boldsymbol{k}}_{1}\right) Y_{l_{15}, m_{15}}\left(\hat{\boldsymbol{k}}_{2}\right),
\end{aligned}
$$

with

$$
\begin{aligned}
d_{l_{5}, l_{14}, l_{15}}\left(k_{1}, k_{2} ; r_{1}, r_{2}\right)= & 2 \pi(-2 i)^{l_{5}} \int_{-1}^{1} d y \frac{\Gamma\left(1-i / 2 k_{3}\right)}{e^{\pi / 4 k_{3}}} \\
& \times \frac{\Gamma\left(l_{5}+i / 2 k_{3}\right)}{\Gamma\left(i / 2 k_{3}\right)} g_{l_{5}, l_{14}}\left(k_{3} ; r_{1}, r_{2}\right) P_{l_{15}}(y),
\end{aligned}
$$

where $y=\hat{\boldsymbol{k}}_{1} \cdot \hat{\boldsymbol{k}}_{2}$. We will return to further consideration of the expressions for $g_{l_{5}, l_{14}}\left(k_{3} ; r_{1}, r_{2}\right)$ and $d_{l_{5}, l_{14}, l_{15}}\left(k_{1}, k_{2} ; r_{1}, r_{2}\right)$ later. Meanwhile, collecting our results we have

$$
\begin{aligned}
& \Psi_{\boldsymbol{k}_{1}, \boldsymbol{k}_{2}}^{(f)-}\left(\boldsymbol{r}_{1}, \boldsymbol{r}_{2}\right)=\sum_{\substack{l_{3}, l_{4}, l_{5}, l_{14}, l_{15}, m_{3}, m_{4}, m_{5}, m_{14}, m_{15}}} \hat{l}_{3}^{2} \hat{l}_{4}^{2} i^{l_{3}+l_{4}} e^{-i \sigma_{l_{3}}\left(k_{1}\right)-i \sigma_{l_{4}}\left(k_{2}\right)} \varphi_{l_{3}}\left(k_{1}, r_{1}\right) \varphi_{l_{4}}\left(k_{2}, r_{2}\right) d_{l_{5}, l_{14}, l_{15}}\left(k_{1}, k_{2} ; r_{1}, r_{2}\right) \\
& \times \sum_{\substack{l_{6}, l_{8}, m_{6}, m_{8}}} \frac{(-1)^{l_{7}+l_{9}}}{2^{l_{8}+l_{9}}} \frac{r_{1}^{l_{6}} r_{2}^{l_{7}} k_{1}^{l_{8}} k_{2}^{l_{9}}}{\left[\left(2 l_{6}\right) !\left(2 l_{7}\right) !\left(2 l_{8}\right) !\left(2 l_{9}\right) !\right]^{1 / 2}} C_{l_{6} m_{6} m_{7} l_{7} m_{7}}^{l_{7}} C_{l_{8} m_{8} l_{9} l_{9} m_{9}} \sum_{\substack{l_{10}, l_{11}, l_{12}, l_{13} \\
m_{10}, m_{11}, m_{12}, m_{13}}} \frac{1}{\hat{l}_{10} \hat{l}_{11} \hat{l}_{12} \hat{l}_{13}} \\
& \times C_{l_{3} m_{3} l_{6} m_{6}}^{l_{10} m_{10}} C_{l_{3} 0 l_{6} 0}^{l_{110} 0} C_{l_{4} m_{4} l_{7} m_{7}}^{l_{11} m_{11}} C_{l_{4} l_{7} 0}^{l_{11} 0} C_{l_{3} m_{3} l_{8} m_{8}}^{l_{12} m_{12}} C_{l_{3} 0 l_{8} 0}^{l_{120} 0} C_{l_{4} m_{4} l_{9} m_{9} m_{9} m_{13}}^{l_{1}} C_{l_{4} 0 l_{9} 0}^{l_{13} 0} Y_{l_{10}, m_{10}}\left(\hat{\boldsymbol{r}}_{1}\right) Y_{l_{14},-m_{14}}\left(\hat{\boldsymbol{r}}_{1}\right) \\
& \times(-1)^{m_{14}} Y_{l_{11}, m_{11}}\left(\hat{\boldsymbol{r}}_{2}\right) Y_{l_{14}, m_{14}}\left(\hat{\boldsymbol{r}}_{2}\right) Y_{l_{12}, m_{12}}^{*}\left(\hat{\boldsymbol{k}}_{1}\right) Y_{l_{15}, m_{15}}^{*}\left(\hat{\boldsymbol{k}}_{1}\right) Y_{l_{13}, m_{13}}^{*}\left(\hat{\boldsymbol{k}}_{2}\right) Y_{l_{15},-m_{15}}^{*}\left(\hat{\boldsymbol{k}}_{2}\right)(-1)^{m_{15}} \text {. }
\end{aligned}
$$

After some momentum algebra we get

$$
\begin{aligned}
& \Psi_{k_{1}, k_{2}}^{(f)-}\left(\boldsymbol{r}_{1}, \boldsymbol{r}_{2}\right)=\frac{1}{(4 \pi)^{2}} \sum_{\substack{l_{3}, l_{4}, l_{5}, l_{14}, l_{15}, m_{3}, m_{4}, m_{5}, m_{14}, m_{15}}} \hat{l}_{3}^{2} \hat{l}_{4}^{2} l^{l_{3}+l_{4}} e^{-i \sigma_{l_{3}}\left(k_{1}\right)-i \sigma_{l_{4}}\left(k_{2}\right)} \varphi_{l_{3}}\left(k_{1}, r_{1}\right) \varphi_{l_{4}}\left(k_{2}, r_{2}\right) d_{l_{5}, l_{14}, l_{15}}\left(k_{1}, k_{2} ; r_{1}, r_{2}\right) \\
& \times \sum_{\substack{l_{6}, l_{8}, m_{6}, m_{8}}} \frac{(-1)^{l_{7}+l_{9}}}{2^{l_{8}+l_{9}}} \frac{r_{1}^{l_{6}} r_{2}^{l_{2}} k_{1}^{l_{8}} k_{2}^{l_{9}}}{\left[\left(2 l_{6}\right) !\left(2 l_{7}\right) !\left(2 l_{8}\right) !\left(2 l_{9}\right) !\right]^{1 / 2}} \sum_{\substack{l_{10}, l_{11}, l_{12}, l_{13}, m_{10}, m_{11}, m_{12}, m_{13}}} \frac{1}{\hat{l}_{10} \hat{l}_{11} \hat{l}_{12} \hat{l}_{13}} C_{l_{6} m_{6} l_{7} m_{7}} C_{l_{8} m_{8} l_{9} m_{9} m_{9}}
\end{aligned}
$$

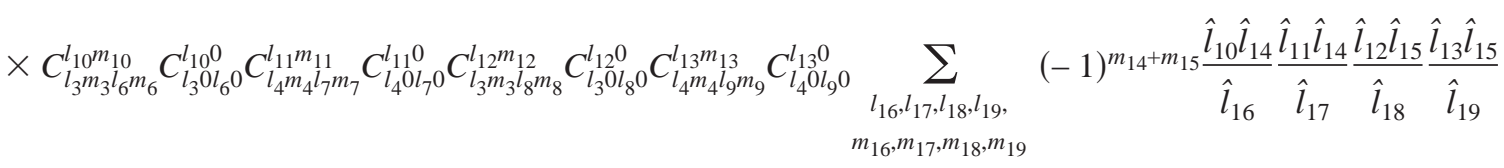

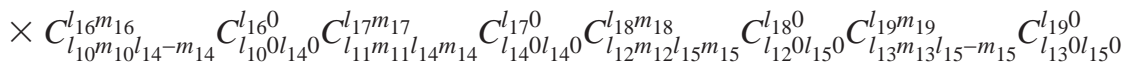

$$
\begin{aligned}
& \times \sum_{L, M} C_{l_{16} m_{16}{ }^{\prime \prime} l_{17} m_{17}} \mathcal{Y}_{l_{16}, l_{17}, L, M}\left(\hat{\boldsymbol{r}}_{1}, \hat{\boldsymbol{r}}_{2}\right) \sum_{L^{\prime}, M^{\prime}} C_{l_{18} L^{\prime} M_{18} l_{19} m_{19}} \mathcal{Y}_{l_{18}, l_{19}, L^{\prime}, M^{\prime}}^{*}\left(\hat{\boldsymbol{k}}_{1}, \hat{\boldsymbol{k}}_{2}\right),
\end{aligned}
$$

where we introduced bipolar harmonics according to 


$$
Y_{l_{1}, m_{1}}\left(\hat{\boldsymbol{r}}_{1}\right) Y_{l_{2}, m_{2}}\left(\hat{\boldsymbol{r}}_{2}\right)=\sum_{L, M} C_{l_{1} m_{1} l_{2} m_{2}}^{L M} \mathcal{Y}_{l_{1}, l_{2}, L, M}\left(\hat{\boldsymbol{r}}_{1}, \hat{\boldsymbol{r}}_{2}\right),
$$

which is easily obtained from definition (88). Performing the summation over projections of the angular momenta we get

$$
\begin{aligned}
& \Psi_{k_{1}, \boldsymbol{k}_{2}}^{(f)-}\left(\boldsymbol{r}_{1}, \boldsymbol{r}_{2}\right)=\frac{1}{(4 \pi)^{2}} \sum_{l_{3}, l_{4}, l_{5}, l_{14}, l_{15}} \hat{l}_{3}^{2} \hat{l}_{4}^{2} \hat{l}_{5}^{2} \hat{l}_{14}^{2} \hat{l}_{15}^{2} l^{2} l^{+}+l_{4} e^{-i \sigma_{l_{3}}\left(k_{1}\right)-i \sigma_{l_{4}}\left(k_{2}\right)} \varphi_{l_{3}}\left(k_{1}, r_{1}\right) \varphi_{l_{4}}\left(k_{2}, r_{2}\right) d_{l_{5}, l_{14}, l_{15}}\left(k_{1}, k_{2} ; r_{1}, r_{2}\right) \\
& \times \sum_{l_{6}, l_{8}} \frac{1}{2^{l_{8}+l_{9}}} \frac{r_{1}^{l_{6}} r_{2}^{l_{7}} k_{1}^{l_{8}} k_{2}^{l_{9}}}{\left[\left(2 l_{6}\right) !\left(2 l_{7}\right) !\left(2 l_{8}\right) !\left(2 l_{9}\right) !\right]^{1 / 2}} \sum_{l_{10}, l_{11}, l_{12}, l_{13}} \hat{l}_{10} \hat{l}_{11} \hat{l}_{12} \hat{l}_{13} C_{l_{3} 0 l_{6} 0}^{l_{11} 0} C_{l_{4} 0 l_{7} 0}^{l_{11} 0} C_{l_{3} 0 l_{8} 0}^{l_{12} 0} C_{l_{4} 0 l_{9} 0}^{l_{13} 0} \\
& \times \sum_{l_{16}, l_{17}, l_{18}, l_{19}} C_{l_{10} 0 l_{14} 0}^{l_{16} 0} C_{l_{11} l_{17} 0}^{l_{14} 0} C_{l_{12} l_{12} 0}^{l_{15} 0} C_{l_{13}}^{l_{19} 0} l_{150} \sum_{L, M}(-1)^{l_{17}-l_{19}}\left\{\begin{array}{ccc}
l_{14} & l_{16} & l_{10} \\
L & l_{11} & l_{17}
\end{array}\right\}\left\{\begin{array}{cccc}
- & l_{5} & l_{6} & l_{7} \\
L & - & l_{10} & l_{11} \\
l_{13} & l_{9} & - & l_{4} \\
l_{12} & l_{8} & l_{3} & -
\end{array}\right\}\left\{\begin{array}{ccc}
l_{15} & l_{18} & l_{12} \\
L & l_{13} & l_{19}
\end{array}\right\} \\
& \times \mathcal{Y}_{l_{16}, l_{17}, L, M}\left(\hat{\boldsymbol{r}}_{1}, \hat{\boldsymbol{r}}_{2}\right) \mathcal{Y}_{l_{18}, l_{19}, L, M}^{*}\left(\hat{\boldsymbol{k}}_{1}, \hat{\boldsymbol{k}}_{2}\right),
\end{aligned}
$$

where the braces denote a $6 j$ symbol and a $12 j$ symbol of the second kind, respectively. Inserting Eq. (107) into Eq. (91) we arrive at the final result for the radial components of the asymptotic wave function we are interested in:

$$
\mathcal{R}_{\lambda, \lambda^{\prime}}^{-}\left(k_{1}, k_{2} ; r_{1}, r_{2}\right)=\widetilde{\mathcal{R}}_{l_{1}, l_{2}, l_{1}^{\prime}, l_{2}^{\prime}, L}^{-}\left(k_{1}, k_{2} ; r_{1}, r_{2}\right) \delta_{L L^{\prime}} \delta_{M M^{\prime}},
$$

with the covariant part given as

$$
\begin{aligned}
\tilde{\mathcal{R}}_{l_{1}, l_{2}, l_{1}^{\prime}, l_{2}^{\prime}, L}^{-}\left(k_{1}, k_{2} ; r_{1}, r_{2}\right)= & \sum_{l_{3}, l_{4}} i^{l_{3}+l_{4}} e^{-i \sigma_{l_{3}}\left(k_{1}\right)-i \sigma_{l_{4}}\left(k_{2}\right)} \\
& \times \varphi_{l_{3}}\left(k_{1}, r_{1}\right) \varphi_{l_{4}}\left(k_{2}, r_{2}\right) \\
& \times f_{l_{1}, l_{2}, l_{1}, l_{1}, l_{2}^{\prime}, L}\left(k_{1}, k_{2} ; r_{1}, r_{2}\right) .
\end{aligned}
$$

For convenience, in the equation above we introduced the shorthand notation

$$
\begin{aligned}
& f_{l_{1}, l_{2}, l_{1}^{\prime}, l_{2}^{\prime}, L}^{l_{2} l_{l}}\left(k_{1}, k_{2} ; r_{1}, r_{2}\right)=\frac{1}{(4 \pi)^{2}} \sum_{l_{5}, l_{14}, l_{15}} \hat{l}_{3}^{2} \hat{l}_{4}^{2} \hat{l}_{5}^{2} \hat{l}_{14}^{2} \hat{l}_{15}^{2} d_{l_{5}, l_{14}, l_{15}}\left(k_{1}, k_{2} ; r_{1}, r_{2}\right) \sum_{l_{6}, l_{8}} \frac{1}{2^{l_{8}+l_{9}}} \frac{r_{1}^{l_{6}} r_{2}^{l_{7}} k_{1}^{l_{8}} k_{2}^{l_{9}}}{\left[\left(2 l_{6}\right) !\left(2 l_{7}\right) !\left(2 l_{8}\right) !\left(2 l_{9}\right) !\right]^{1 / 2}}
\end{aligned}
$$

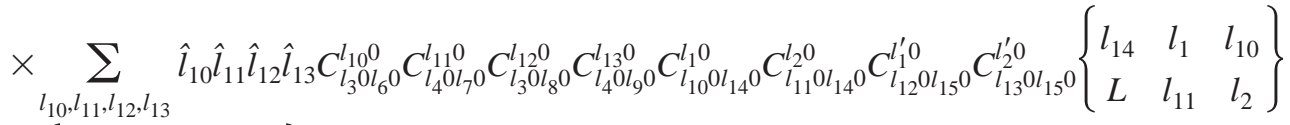

$$
\begin{aligned}
& \times\left\{\begin{array}{cccc}
- & l_{5} & l_{6} & l_{7} \\
L & - & l_{10} & l_{11} \\
l_{13} & l_{9} & - & l_{4} \\
l_{12} & l_{8} & l_{3} & -
\end{array}\right\}\left\{\begin{array}{ccc}
l_{15} & l_{1}^{\prime} & l_{12} \\
L & l_{13} & l_{2}^{\prime}
\end{array}\right\} .
\end{aligned}
$$

We draw the reader's attention to the fact that we did not assume in our derivation the property that the expansion coefficients were diagonal in $L$ and $M$. It is a test of the correctness of our angular momentum algebra that this anticipated result emerges.

We finally return to giving useful expressions for calculating the $g_{l_{5}, l_{14}}\left(k_{3} ; r_{1}, r_{2}\right)$ and $d_{l_{5}, l_{14}, l_{15}}\left(k_{1}, k_{2} ; r_{1}, r_{2}\right)$. After some algebra we get

$$
\begin{aligned}
g_{l_{5}, l_{14}}\left(k_{3} ; r_{1}, r_{2}\right)= & 2 \pi \sum_{n=0}^{\left[l_{14} / 2\right]} a_{n} \sum_{N=0}^{l_{14}-2 n} b_{N} \frac{\left(r_{1}^{2}+r_{2}^{2}\right)^{l_{14}-2 n-N}}{\left(2 r_{1} r_{2}\right)^{l_{14}-2 n+1}} \\
& \times\left[D_{l_{5}, N}\left(k_{3}, r_{1}+r_{2}\right)-D_{l_{5}, N}\left(k_{3},\left|r_{1}-r_{2}\right|\right)\right],
\end{aligned}
$$

where 


$$
\begin{gathered}
a_{n}=\frac{(-1)^{n}}{2^{l_{14}}} \frac{\left(2 l_{14}-2 n\right) !}{n !\left(l_{14}-n\right) !}, \\
b_{N}=\frac{(-1)^{N}}{(N+1) !} \frac{1}{\left(l_{14}-2 n-N\right) !},
\end{gathered}
$$

and $\left[l_{14} / 2\right]$ is the integer part of $l_{14} / 2$. The function $D_{l_{5}, N}\left(k_{3}, r\right)$ is given by

$$
\begin{aligned}
& D_{l_{5}, N}\left(k_{3}, r\right) \\
& \quad=r^{2 N+2}{ }_{2} F_{2}\left(2 N+2, l_{5}+i / 2 k_{3} ; 2 N+3,2 l_{5}+2 ;-2 i k_{3} r\right) .
\end{aligned}
$$

With this result the remaining integral can be transformed to

$$
\begin{aligned}
d_{l_{5}, l_{14}, l_{15}}\left(k_{1}, k_{2} ; r_{1}, r_{2}\right)= & (2 \pi)^{2}(-2 i)^{l_{5}} \sum_{n=0}^{\left[l_{1} / 2\right]} a_{n} \sum_{N=0}^{l_{14}-2 n} b_{N} \frac{\left(r_{1}^{2}+r_{2}^{2}\right)^{l_{14}-2 n-N}}{\left(2 r_{1} r_{2}\right)^{l_{14}-2 n+1}} \frac{1}{k_{1} k_{2}} \int_{\left|k_{1}-k_{2}\right|}^{k_{1}+k_{2}} d k k \frac{\Gamma(1-i / 2 k)}{e^{\pi / 4 k}} \frac{\Gamma\left(l_{5}+i / 2 k\right)}{\Gamma(i / 2 k)} P_{l_{15}}\left(\frac{k_{1}^{2}+k_{2}^{2}-k^{2}}{2 k_{1} k_{2}}\right) \\
& \times\left[D_{l_{5}, N}\left(k, r_{1}+r_{2}\right)-D_{l_{5}, N}\left(k,\left|r_{1}-r_{2}\right|\right)\right] .
\end{aligned}
$$

Thus we established that for our particular physical system the general expansion (90) can be written as

$$
\begin{aligned}
\Psi_{\boldsymbol{k}_{1}, \boldsymbol{k}_{2}}^{(f)-}\left(\boldsymbol{r}_{1}, \boldsymbol{r}_{2}\right)= & \sum_{l_{1}, l_{2}, l_{1}^{\prime}, l_{2}^{\prime}, L, M} \widetilde{\mathcal{R}}_{l_{1}, l_{2}, l_{1}^{\prime}, l_{2}^{\prime}, L}^{-}\left(k_{1}, k_{2} ; r_{1}, r_{2}\right) \\
& \times \mathcal{Y}_{l_{1}^{\prime}, l_{2}^{\prime}, L, M}^{*}\left(\hat{\boldsymbol{k}}_{1}, \hat{\boldsymbol{k}}_{2}\right) \mathcal{Y}_{l_{1}, l_{2}, L, M}\left(\hat{\boldsymbol{r}}_{1}, \hat{\boldsymbol{r}}_{2}\right),
\end{aligned}
$$

with the expansion coefficients given by Eq. (109) being rotationally invariant.

\section{B. Partial waves of $\Phi_{c}^{+}\left(r_{1}, r_{2}\right)$}

We next turn to the critical task of finding the asymptotic form of the expansion coefficients $\mathcal{R}_{l_{1}, l_{2}, L, M}^{+}\left(r_{1}, r_{2}\right)$ defined by Eq. (89). As mentioned earlier, for this we use a fundamental asymptotic relationship established recently [16,17], which for the continuum part of the scattered wave function reads as

$$
\Phi_{c}^{+}\left(\boldsymbol{r}_{1}, \boldsymbol{r}_{2}\right)=\int \frac{d \boldsymbol{k}_{1}^{\prime}}{(2 \pi)^{3}} \frac{d \boldsymbol{k}_{2}^{\prime}}{(2 \pi)^{3}} \frac{T\left(\boldsymbol{k}_{1}^{\prime}, \boldsymbol{k}_{2}^{\prime}\right) \Psi_{\boldsymbol{k}_{1}^{\prime}, \boldsymbol{k}_{2}^{\prime}}^{(f)-}\left(\boldsymbol{r}_{1}, \boldsymbol{r}_{2}\right)}{E-k_{1}^{\prime 2} / 2-k_{2}^{\prime 2} / 2+i 0}
$$

In Refs. [16,17] this relationship was used to obtain $\Phi_{c}^{+}$from the asymptotic form of $\Psi^{(f)-}$. Here we first expand Eq. (117) in partial waves and then calculate the resulting integrals by means of asymptotic methods.

Before proceeding further we want to make the following remark. Since $r_{1}, r_{2} \rightarrow \infty$, the integral in Eq. (117) is extremely oscillatory with the strongest oscillations coming from the term $e^{i \boldsymbol{k}_{1} \cdot \boldsymbol{r}_{1}+i \boldsymbol{k}_{2} \cdot \boldsymbol{r}_{2}}$ of the wave function $\Psi_{\boldsymbol{k}_{1}, \boldsymbol{k}_{2}}^{(f)-}\left(\boldsymbol{r}_{1}, \boldsymbol{r}_{2}\right)$. When we calculated Eq. (117) without partial-wave expansion (see $[16,17]$ ) we observed that the dominant contribution came from a small neighborhood of a stationary-phase point of the term $e^{i \boldsymbol{k}_{1} \cdot \boldsymbol{r}_{1}+i \boldsymbol{k}_{2} \cdot \boldsymbol{r}_{2}}$. The only contribution to the integral came from the following directions [recall Eq. (31)]:

$$
e^{i \boldsymbol{k}_{1} \cdot \boldsymbol{r}_{1}+i \boldsymbol{k}_{2} \cdot \boldsymbol{r}_{2}} \stackrel{r_{1}, r_{2} \rightarrow \infty}{\propto} \delta\left(\hat{\boldsymbol{k}}_{1}-\hat{\boldsymbol{r}}_{1}\right) \delta\left(\hat{\boldsymbol{k}}_{2}-\hat{\boldsymbol{r}}_{2}\right),
$$

which can be written in partial waves as

$$
\begin{aligned}
& \delta\left(\hat{\boldsymbol{k}}_{1}-\hat{\boldsymbol{r}}_{1}\right) \delta\left(\hat{\boldsymbol{k}}_{2}-\hat{\boldsymbol{r}}_{2}\right) \\
& =\sum_{l_{1}, m_{1}} Y_{l_{1}, m_{1}}^{*}\left(\hat{\boldsymbol{k}}_{1}\right) Y_{l_{1}, m_{1}}\left(\hat{\boldsymbol{r}}_{1}\right) \sum_{l_{2}, m_{2}} Y_{l_{2}, m_{2}}^{*}\left(\hat{\boldsymbol{k}}_{2}\right) Y_{l_{2}, m_{2}}\left(\hat{\boldsymbol{r}}_{2}\right) \\
& =\sum_{l_{1}, l_{2}, L^{\prime}, M^{\prime}} \sum_{l_{1} m_{1} l_{2} m_{2}}^{L^{\prime} M^{\prime}} \mathcal{Y}_{l_{1}, l_{2}, L^{\prime}, M^{\prime}}^{*}\left(\hat{\boldsymbol{k}}_{1}, \hat{\boldsymbol{k}}_{2}\right) \\
& m_{1}, m_{2} \\
& \times \sum_{L, M} C_{l_{1} m_{1} l_{2} m_{2}}^{L M} \mathcal{Y}_{l_{1}, l_{2}, L, M}\left(\hat{\boldsymbol{r}}_{1}, \hat{\boldsymbol{r}}_{2}\right) \\
& =\sum_{l_{1}, l_{2}, L, M} \mathcal{Y}_{l_{1}, l_{2}, L, M}^{*}\left(\hat{\boldsymbol{k}}_{1}, \hat{\boldsymbol{k}}_{2}\right) \mathcal{Y}_{l_{1}, l_{2}, L, M}\left(\hat{\boldsymbol{r}}_{1}, \hat{\boldsymbol{r}}_{2}\right) .
\end{aligned}
$$

This leads to the conclusion that whenever we are calculating the partial-wave expansion of Eq. (117) for $r_{1}, r_{2} \rightarrow \infty$ utilizing Eq. (116) we should only consider contributions for $l_{1}^{\prime}$ $=l_{1}$ and $l_{2}^{\prime}=l_{2}$. Other values of $l_{1}^{\prime}$ and $l_{2}^{\prime}$ do not contribute to the integral in the regions where the stationary-phase methods apply.

Let us now expand the ionization amplitude as usual in the standard manner:

$$
T\left(\boldsymbol{k}_{1}, \boldsymbol{k}_{2}\right)=\sum_{l_{1}, l_{2}, L, M} \mathcal{T}_{l_{1}, l_{2}, L, M}\left(k_{1}, k_{2}\right) \mathcal{Y}_{l_{1}, l_{2}, L, M}^{*}\left(\hat{\boldsymbol{k}}_{1}, \hat{\boldsymbol{k}}_{2}\right),
$$

where $\mathcal{T}_{l_{1}, l_{2}, L, M}$ are the partial ionization amplitudes defined as

$$
\mathcal{T}_{l_{1}, l_{2}, L, M}\left(k_{1}, k_{2}\right)=\int d \hat{\boldsymbol{k}}_{1} d \hat{\boldsymbol{k}}_{2} T\left(\boldsymbol{k}_{1}, \boldsymbol{k}_{2}\right) \mathcal{Y}_{l_{1}, l_{2}, L, M}^{*}\left(\hat{\boldsymbol{k}}_{1}, \hat{\boldsymbol{k}}_{2}\right)
$$


Then using expansions (116) and (120) in (117) and keeping only the terms capable of contributing to the result we get

$$
\begin{aligned}
\Phi_{c}^{+}\left(\boldsymbol{r}_{1}, \boldsymbol{r}_{2}\right)= & \sum_{l_{1}, l_{2}, L, M} \int \frac{d k_{1}^{\prime} k_{1}^{\prime 2}}{(2 \pi)^{3}} \frac{d k_{2}^{\prime} k_{2}^{\prime 2}}{(2 \pi)^{3}} \\
& \times \frac{\mathcal{T}_{l_{1}, l_{2}, L, M}\left(k_{1}^{\prime}, k_{2}^{\prime}\right) \tilde{\mathcal{R}}_{l_{1}, l_{2}, l_{1}, l_{2}, L}^{-}\left(k_{1}^{\prime}, k_{2}^{\prime} ; r_{1}, r_{2}\right)}{E-k_{1}^{\prime 2} / 2-k_{2}^{\prime 2} / 2+i 0} \\
& \times \mathcal{Y}_{l_{1}, l_{2}, L, M}\left(\hat{\boldsymbol{r}}_{1}, \hat{\boldsymbol{r}}_{2}\right) .
\end{aligned}
$$

From this equation, by direct comparison with the definition (87) of the partial-wave expansion, we see that the asymptotic form in the ionization sector (in the following, $\mathcal{R}_{l_{1}, l_{2}, L, M}$ will refer only to the $\Omega_{c}$ part of the radial wave function as we are not interested in its two-fragment parts) is

$$
\begin{aligned}
\mathcal{R}_{l_{1}, l_{2}, L, M}^{+}\left(r_{1}, r_{2}\right)= & \int \frac{d k_{1}^{\prime} k_{1}^{\prime 2}}{(2 \pi)^{3}} \frac{d k_{2}^{\prime} k_{2}^{\prime 2}}{(2 \pi)^{3}} \\
& \times \frac{\mathcal{T}_{l_{1}, l_{2}, L, M}\left(k_{1}^{\prime}, k_{2}^{\prime}\right) \tilde{\mathcal{R}}_{l_{1}, l_{2}, l_{1}, l_{2}, L}^{-}\left(k_{1}^{\prime}, k_{2}^{\prime} ; r_{1}, r_{2}\right)}{E-k^{\prime 2}{ }_{1} / 2-k_{2}^{\prime 2} / 2+i 0} .
\end{aligned}
$$

To obtain a useful result we still need to evaluate the integral. The explicit form of $\widetilde{\mathcal{R}}_{l_{1}, l_{2}, l_{1}, l_{2}, L}^{-}$in $\Omega_{c}$ has been given above in Eq. (109). The function $\varphi_{l}(k, r)$ has the asymptotic behavior

$$
\varphi_{l}(k, r) \stackrel{r \rightarrow \infty}{\sim} \frac{4 \pi}{k r} \sin \left[k r+(1 / k) \ln (2 k r)-l \pi / 2+\sigma_{l}(k)\right] .
$$

Therefore, substituting Eq. (109) in Eq. (123) and evaluating the integral over $k_{1}^{\prime}$ taking advantage of the simple pole singularity (ps) of the integrand we get, in the leading order,

$$
\begin{aligned}
& \mathcal{R}_{l_{1}, l_{2}, L, M}^{+}\left(r_{1}, r_{2}\right)=\sum_{l_{3}, l_{4}} \int \frac{d k_{1}^{\prime} k_{1}^{\prime 2}}{(2 \pi)^{3}} \frac{d k_{2}^{\prime} k_{2}^{\prime 2}}{(2 \pi)^{3}} \frac{\mathcal{T}_{l_{1}, l_{2}, L, M}\left(k_{1}^{\prime}, k_{2}^{\prime}\right) i^{l_{3}} e^{-i \sigma_{l_{3}}\left(k_{1}^{\prime}\right)} \varphi_{l_{3}}\left(k_{1}^{\prime}, r_{1}\right)}{E-k_{1}^{\prime 2} / 2-k_{2}^{\prime 2} / 2+i 0} i^{l_{4}} e^{-i \sigma_{l_{4}}\left(k_{2}^{\prime}\right)} \varphi_{l_{4}}\left(k_{2}^{\prime}, r_{2}\right) f_{l_{1}, l_{2}, l_{1}, l_{2}, L}^{l_{3}, l_{4}}\left(k_{1}^{\prime}, k_{2}^{\prime} ; r_{1}, r_{2}\right) \\
& =\frac{1}{i r_{1} l_{3}, l_{4}} \int \frac{d k_{1}^{\prime} k^{\prime}}{(2 \pi)^{2}} \frac{d k_{2}^{\prime} k_{2}^{\prime 2}}{(2 \pi)^{3}} \frac{\mathcal{T}_{l_{1}, l_{2}, L, M}\left(k_{1}^{\prime}, k_{2}^{\prime}\right)}{E-k_{1}^{\prime 2} / 2-k_{2}^{\prime 2} / 2+i 0} i^{i l_{3}} e^{-i \sigma_{l_{3}}\left(k_{1}^{\prime}\right)}\left(e^{i k_{1}^{\prime} r_{1}+i / k_{1}^{\prime} \ln \left(2 k_{1}^{\prime} r_{1}\right)-i l_{3} \pi / 2+i \sigma_{l_{3}}\left(k_{1}^{\prime}\right)}\right. \\
& \left.-e^{-i k_{1}^{\prime} r_{1}-i / k_{1}^{\prime} \ln \left(2 k_{1}^{\prime} r_{1}\right)+i l_{3} \pi / 2-i \sigma_{l_{3}}\left(k_{1}^{\prime}\right)}\right) i^{l_{4}} e^{-i \sigma_{l_{4}}\left(k_{2}^{\prime}\right)} \varphi_{l_{4}}\left(k_{2}^{\prime}, r_{2}\right) f_{l_{1}, l_{2}, l_{1}, l_{2}, L}^{l_{3}, l_{4}}\left(k_{1}^{\prime}, k_{2}^{\prime} ; r_{1}, r_{2}\right)
\end{aligned}
$$

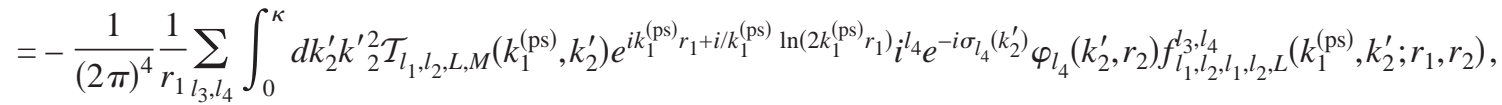

where $k_{1}^{(\mathrm{ps})}=\left(\kappa^{2}-k_{2}^{\prime 2}\right)^{1 / 2}$. Then using Eq. (124) leads to

$$
\begin{aligned}
\mathcal{R}_{l_{1}, l_{2}, L, M}^{+}\left(r_{1}, r_{2}\right)= & -\frac{1}{(2 \pi)^{3}} \frac{1}{i r_{1} r_{2}} \sum_{l_{3}, l_{4}} \int_{0}^{\kappa} d k_{2}^{\prime} k^{\prime}{ }_{2} \mathcal{T}_{l_{1}, l_{2}, L, M}\left(k_{1}^{(\mathrm{ps})}, k_{2}^{\prime}\right) e^{i k_{1}^{(\mathrm{ps})} r_{1}+i l k_{1}^{(\mathrm{ps})}} \ln \left(2 k_{1}^{(\mathrm{ps})} r_{1}\right) i_{4} l_{4} e^{-i \sigma_{l_{4}}\left(k_{2}^{\prime}\right)}\left(e^{i k_{2}^{\prime} r_{2}+i l k_{2}^{\prime} \ln \left(2 k_{2}^{\prime} r_{2}\right)-i l_{4} \pi / 2+i \sigma_{l_{4}}\left(k_{2}^{\prime}\right)}\right. \\
& \left.-e^{-i k_{2}^{\prime} r_{2}-i / k_{2}^{\prime} \ln \left(2 k_{2}^{\prime} r_{2}\right)+i l_{4} \pi / 2-i \sigma_{l_{4}}\left(k_{2}^{\prime}\right)}\right) f_{l_{1}, l_{2}, l_{1}, l_{2}, L}^{l_{3}, l_{1}}\left(k_{1}^{(\mathrm{ps})}, k_{2}^{\prime} ; r_{1}, r_{2}\right) .
\end{aligned}
$$

The first term of this highly oscillatory integral has a single stationary point at $k_{2}^{\prime}=\kappa r_{2} / R \equiv \kappa \sin \alpha$, where now $k_{1}^{(\mathrm{ps})}=\kappa r_{1} / R$ $=\kappa \cos \alpha$. The second term does not have any stationary points; therefore it does not contribute. Thus evaluating the remaining integral we finally have

$$
\begin{aligned}
\mathcal{R}_{l_{1}, l_{2}, L, M}^{+}\left(r_{1}, r_{2}\right)= & \frac{1}{(2 \pi)^{5 / 2}} \mathcal{T}_{l_{1}, l_{2}, L, M}(\kappa \cos \alpha, \kappa \sin \alpha) \frac{\kappa^{3 / 2}}{R^{5 / 2}} \exp \left(i \kappa R+\frac{i \ln \left(2 \kappa R \cos ^{2} \alpha\right)}{\kappa \cos \alpha}+\frac{i \ln \left(2 \kappa R \sin ^{2} \alpha\right)}{\kappa \sin \alpha}+\frac{i \pi}{4}\right) \\
& \times \tau_{l_{1}, l_{2}, L}(\kappa \cos \alpha, \kappa \sin \alpha ; R \cos \alpha, R \sin \alpha),
\end{aligned}
$$


where

$$
\begin{aligned}
& \tau_{l_{1}, l_{2}, L}(\kappa \cos \alpha, \kappa \sin \alpha ; R \cos \alpha, R \sin \alpha) \\
& \quad=\sum_{l_{3}, l_{4}} f_{l_{1}, l_{2}, l_{1}, l_{2}, L}^{l_{3}, l_{4}}(\kappa \cos \alpha, \kappa \sin \alpha ; R \cos \alpha, R \sin \alpha) .
\end{aligned}
$$

A crucial feature of these asymptotic wave functions is that they have a form where the partial ionization amplitudes are factorized. In the following section we will demonstrate how to obtain the partial-wave ionization amplitudes from the partial-wave radial functions derived here.

\section{INTEGRAL REPRESENTATION FOR THE PARTIAL IONIZATION AMPLITUDES}

In order to establish an integral representation for the partial ionization amplitudes we need, in addition to the partialwave expansion of the scattered wave, a similar expansion for our auxiliary wave function $\Psi^{(2 \mathrm{C})}$ - . Therefore we expand it according to

$$
\begin{aligned}
\Psi_{\boldsymbol{k}_{1}, \boldsymbol{k}_{2}}^{(2 \mathrm{C})-}\left(\boldsymbol{r}_{1}, \boldsymbol{r}_{2}\right)= & \sum_{l_{1}, l_{2}, L, M} \mathcal{R}_{l_{1}, l_{2}}^{(2 \mathrm{C})-}\left(k_{1}, k_{2} ; r_{1}, r_{2}\right) \\
& \times \mathcal{Y}_{l_{1}, l_{2}, L, M}^{*}\left(\hat{\boldsymbol{k}}_{1}, \hat{\boldsymbol{k}}_{2}\right) \mathcal{Y}_{l_{1}, l_{2}, L, M}\left(\hat{\boldsymbol{r}}_{1}, \hat{\boldsymbol{r}}_{2}\right),
\end{aligned}
$$

where the radial coefficients $\mathcal{R}_{l_{1}, l_{2}}^{(2 \mathrm{C})-}\left(r_{1}, r_{2}\right)$ of this expansion are given by

$$
\mathcal{R}_{l_{1}, l_{2}}^{(2 \mathrm{C})-}\left(r_{1}, r_{2}\right)=i^{l_{1}+l_{2}} e^{-i \sigma_{l_{1}}\left(k_{1}\right)-i \sigma_{l_{2}}\left(k_{2}\right)} \varphi_{l_{1}}\left(k_{1}, r_{1}\right) \varphi_{l_{2}}\left(k_{2}, r_{2}\right) .
$$

The expansion (129) with radial coefficients (130) is easily derived by means of expanding the Coulomb wave functions in terms of the ordinary spherical harmonics and coupling the corresponding pair of spherical harmonics into bipolar harmonics. Alternatively, it is also not difficult to obtain it from our general expansion (90) and (91) and (108) and (109)when the electron-electron interaction is turned off.

Now we can write the integral (11) for asymptotically large $R$ as

$$
I\left(\boldsymbol{k}_{1}, \boldsymbol{k}_{2} ; R\right)=\sum_{l_{1}, l_{2}, L, M} \mathcal{I}_{l_{1}, l_{2}, L, M}\left(k_{1}, k_{2} ; R\right) \mathcal{Y}_{l_{1}, l_{2}, L, M}^{*}\left(\hat{\boldsymbol{k}}_{1}, \hat{\boldsymbol{k}}_{2}\right),
$$

where

$$
\begin{aligned}
\mathcal{I}_{l_{1}, l_{2}, L, M}\left(k_{1}, k_{2} ; R\right)= & \frac{1}{2} R^{5} \int_{0}^{\pi / 2} d \alpha \sin ^{2} \alpha \\
& \times \cos ^{2} \alpha\left(\mathcal{R}_{l_{1}, l_{2}}^{(2 \mathrm{C})-*} \frac{\partial \mathcal{R}_{l_{1}, l_{2}, L, M}^{+}}{\partial R}\right. \\
& \left.-\mathcal{R}_{l_{1}, l_{2}, L, M}^{+} \frac{\partial \mathcal{R}_{l_{1}, l_{2}}^{(2 \mathrm{C}) *}}{\partial R}\right)
\end{aligned}
$$

are the partial-wave components of our trial integral. All information about the partial ionization amplitudes is contained in the radial parts $\mathcal{R}_{l_{1}, l_{2}, L, M}^{+}$of the wave function $\Phi_{i}^{+}$. Let us now calculate integral (132).

Since the function $\varphi_{l}(k, r)$ asymptotically behaves according to Eq. (124), for the integral (132) we get

$$
\begin{aligned}
\mathcal{I}_{l_{1}, l_{2}, L, M}\left(k_{1}, k_{2} ; R\right)= & \frac{1}{2} \frac{1}{(2 \pi)^{1 / 2}} \frac{\kappa^{3 / 2}}{i k_{1} k_{2}} R^{1 / 2} \int_{0}^{\pi / 2} d \alpha \sin \alpha \cos \alpha \mathcal{T}_{l_{1}, l_{2}, L, M}(\kappa \cos \alpha, \kappa \sin \alpha) \exp \left[i \sigma_{l_{1}}\left(k_{1}\right)+i \sigma_{l_{2}}\left(k_{2}\right)-i \pi l_{1} / 2-i \pi l_{2} / 2\right] \\
& \times \exp \left(i \kappa R+\frac{i \ln \left(2 \kappa R \cos ^{2} \alpha\right)}{\kappa \cos \alpha}+\frac{i \ln \left(2 \kappa R \sin ^{2} \alpha\right)}{\kappa \sin \alpha}+\frac{i \pi}{4}\right)\left\{( \kappa + k _ { 1 } \operatorname { c o s } \alpha + k _ { 2 } \operatorname { s i n } \alpha ) \operatorname { e x p } \left[-i k_{1} R \cos \alpha\right.\right. \\
& \left.-\left(i / k_{1}\right) \ln \left(2 k_{1} R \cos \alpha\right)+i l_{1} \pi / 2-i \sigma_{l_{1}}\left(k_{1}\right)\right] \exp \left[-i k_{2} R \sin \alpha-\left(i / k_{2}\right) \ln \left(2 k_{2} R \sin \alpha\right)+i l_{2} \pi / 2-i \sigma_{l_{2}}\left(k_{2}\right)\right] \\
& +\left(\kappa-k_{1} \cos \alpha-k_{2} \sin \alpha\right) \exp \left[i k_{1} R \cos \alpha+\left(i / k_{1}\right) \ln \left(2 k_{1} R \cos \alpha\right)-i l_{1} \pi / 2+i \sigma_{l_{1}}\left(k_{1}\right)\right] \exp \left[i k_{2} R \sin \alpha\right. \\
& \left.+\left(i / k_{2}\right) \ln \left(2 k_{2} R \sin \alpha\right)-i l_{2} \pi / 2+i \sigma_{l_{2}}\left(k_{2}\right)\right]-\left(\kappa+k_{1} \cos \alpha-k_{2} \sin \alpha\right) \exp \left[-i k_{1} R \cos \alpha\right. \\
& \left.-\left(i / k_{1}\right) \ln \left(2 k_{1} R \cos \alpha\right)+i l_{1} \pi / 2-i \sigma_{l_{1}}\left(k_{1}\right)\right] \exp \left[i k_{2} R \sin \alpha+\left(i / k_{2}\right) \ln \left(2 k_{2} R \sin \alpha\right)-i l_{2} \pi / 2+i \sigma_{l_{2}}\left(k_{2}\right)\right] \\
& -\left(\kappa-k_{1} \cos \alpha+k_{2} \sin \alpha\right) \exp \left[i k_{1} R \cos \alpha+\left(i / k_{1}\right) \ln \left(2 k_{1} R \cos \alpha\right)-i l_{1} \pi / 2+i \sigma_{l_{1}}\left(k_{1}\right)\right] \exp \left[-i k_{2} R \sin \alpha\right. \\
& \left.\left.-\left(i / k_{2}\right) \ln \left(2 k_{2} R \sin \alpha\right)+i l_{2} \pi / 2-i \sigma_{l_{2}}\left(k_{2}\right)\right]\right\} \tau_{l_{1}, l_{2}, L}(\kappa \cos \alpha, \kappa \sin \alpha ; R \cos \alpha, R \sin \alpha) .
\end{aligned}
$$


The first two terms of this high-oscillatory integral have a stationary point at $\alpha=\arctan \left(k_{2} / k_{1}\right)$, where

$$
\kappa \cos \alpha=k_{1} \quad \text { and } \quad \kappa \sin \alpha=k_{2} .
$$

However, the second term is identically zero at this point. The other two terms do not have any stationary points and therefore they do not contribute to the result. Calculating the remaining term we finally have

$$
\mathcal{I}_{l_{1}, l_{2}, L, M}\left(k_{1}, k_{2} ; R\right)=\mathcal{T}_{l_{1}, l_{2}, L, M}\left(k_{1}, k_{2}\right) \tau_{l_{1}, l_{2}, L}\left(k_{1}, k_{2} ; k_{1} R / \kappa, k_{2} R / \kappa\right)
$$

or

$$
\mathcal{T}_{l_{1}, l_{2}, L, M}\left(k_{1}, k_{2}\right)=\lim _{R \rightarrow \infty} \frac{\mathcal{I}_{l_{1}, l_{2}, L, M}\left(k_{1}, k_{2} ; R\right)}{\tau_{l_{1}, l_{2}, L, M}\left(k_{1}, k_{2} ; k_{1} R / \kappa, k_{2} R / \kappa\right)} .
$$

Thus the knowledge of the radial part of the scattering wave function allows us to represent the partial ionization amplitudes in terms of the partial waves $\mathcal{I}_{l_{1}, l_{2}, L, M}$ of Peterkop's trial integral (6). Note that for practical calculations one would compute

$$
\begin{aligned}
\mathcal{T}_{l_{1}, l_{2}, L, M}\left(k_{1}, k_{2} ; R\right) \equiv & \mathcal{I}_{l_{1}, l_{2}, L, M}\left(k_{1}, k_{2} ; R\right) / \\
& \tau_{l_{1}, l_{2}, L, M}\left(k_{1}, k_{2} ; k_{1} R / \kappa, k_{2} R / \kappa\right)
\end{aligned}
$$

at large $R$ and establish the limit by extrapolation to $R \rightarrow \infty$. In doing so $\mathcal{I}_{l_{1}, l_{2}, L, M}\left(k_{1}, k_{2} ; R\right)$ is calculated from Eq. (132) with $\mathcal{R}_{l_{1}, l_{2}, L, M}^{+}$coming from the solution of the partial-wave Schrödinger equation.

The use of the integral representation (136) is one way of calculating the partial ionization amplitudes. Alternatively one can use the surface-integral forms derived in Sec. IV. Expanding, for instance, the $a c$ form of the ionization amplitude [Eq. (81)] according to Eqs. (120) and (121) we obtain

$$
\begin{aligned}
\mathcal{T}_{l_{1}, l_{2}, L, M}\left(k_{1}, k_{2}\right)= & \frac{1}{2} \lim _{R \rightarrow \infty} R^{5} \int_{0}^{\pi / 2} d \alpha \sin ^{2} \alpha \cos ^{2} \alpha \\
& \times\left(\tilde{\mathcal{R}}_{l_{1}, l_{2}, l_{1}, l_{2}, L}^{-*} \frac{\partial \mathcal{R}_{l_{1}, l_{2}, L, M}^{+}}{\partial R}\right. \\
& \left.-\mathcal{R}_{l_{1}, l_{2}, L, M}^{+} \frac{\partial \widetilde{\mathcal{R}}_{l_{1}, l_{2}, l_{1}, l_{2}, L}^{-*}}{\partial R}\right) .
\end{aligned}
$$

This is how the partial ionization amplitudes are given in their natural form, without the use of an auxiliary function. Numerical calculation of the integral in Eq. (137) directly gives us the partial ionization amplitudes. Again, the wave functions $\mathcal{R}_{l_{1}, l_{2}, L, M}$ come from the solution of the Schrödinger equation.

On the other hand analytical evaluation of the integral in Eq. (137) allows us to establish a very important practical result. To calculate the integral we use Eqs. (109) and (127) and follow the procedure we applied above. Then we arrive at

$$
\begin{aligned}
& \mathcal{T}_{l_{1}, l_{2}, L, M}\left(k_{1}, k_{2}\right) \\
& \quad=\mathcal{T}_{l_{1}, l_{2}, L, M}\left(k_{1}, k_{2}\right) \lim _{R \rightarrow \infty}\left|\tau_{l_{1}, l_{2}, L, M}\left(k_{1}, k_{2} ; k_{1} R / \kappa, k_{2} R / \kappa\right)\right|^{2} .
\end{aligned}
$$

From this we conclude that

$$
\lim _{R \rightarrow \infty}\left|\tau_{l_{1}, l_{2}, L, M}\left(k_{1}, k_{2} ; k_{1} R / \kappa, k_{2} R / \kappa\right)\right| \rightarrow 1 .
$$

Consequently, from Eq. (136) we get

$$
\left|\mathcal{T}_{l_{1}, l_{2}, L, M}\left(k_{1}, k_{2}\right)\right|=\lim _{R \rightarrow \infty}\left|\mathcal{I}_{l_{1}, l_{2}, L, M}\left(k_{1}, k_{2} ; R\right)\right|,
$$

an important relationship, which rigorously proves the validity of the partial-wave-based calculations of the ionization cross sections in the full electron-hydrogen ionization problem using ECS techniques [8-10] (when $R$ is sufficiently large).

\section{APPLICATION TO MODEL PROBLEMS}

In this section we consider some well-known model problems. We present results obtained in our approach for the wave functions and ionization amplitudes readily applicable for practical calculations.

\section{A. Screening model}

Consider a model electron-hydrogen ionization problem where electron $e_{1}$ is completely shielded from the proton by electron $e_{2}$ which is closer to the proton. We call this a "one Coulomb" (1C) model. The asymptotic form of the unscattered wave function $\Psi^{(f)-}$ for this model is given by

$$
\Psi_{\boldsymbol{k}_{1}, \boldsymbol{k}_{2}}^{(1 \mathrm{C})-}\left(\boldsymbol{r}_{1}, \boldsymbol{r}_{2}\right)=e^{i \boldsymbol{k}_{1} \cdot \boldsymbol{r}_{1}+i \boldsymbol{k}_{2} \cdot \boldsymbol{r}_{2}} \psi_{1}\left(\boldsymbol{k}_{2}, \boldsymbol{r}_{2}\right)
$$

The partial waves of this function are

$$
\mathcal{R}_{l_{1}, l_{2}}^{(1 \mathrm{C})-}\left(r_{1}, r_{2}\right)=4 \pi i^{l_{1}+l_{2}} e^{-i \sigma_{l_{2}}\left(k_{2}\right)} j_{l_{1}}\left(k_{1} r_{1}\right) \phi_{l_{2}}\left(k_{2}, r_{2}\right),
$$

where $j_{l}(k r)$ is the spherical Bessel function.

Then using Eqs. (141) and (117) according to [16,17] we get the corresponding scattered wave for ionization to be

$$
\begin{aligned}
\Phi_{c}^{(1 \mathrm{C})+}\left(\boldsymbol{r}_{1}, \boldsymbol{r}_{2}\right)= & \frac{1}{(2 \pi)^{5 / 2}} T^{(1 \mathrm{C})}\left(\kappa \cos \alpha \hat{\boldsymbol{r}}_{1}, \kappa \sin \alpha \hat{\boldsymbol{r}}_{2}\right) \frac{\kappa^{3 / 2}}{R^{5 / 2}} n \\
& \times \exp \left(i \kappa R+\frac{i \ln \left(2 \kappa R \sin ^{2} \alpha\right)}{\kappa \sin \alpha}+\frac{i \pi}{4}\right),
\end{aligned}
$$

where $T^{(1 \mathrm{C})}$ is the ionization amplitude in the $1 \mathrm{C}$ model. This allows us to write the asymptotic form of the partial waves of $\Phi_{c}^{+}$for this model: 


$$
\begin{aligned}
\mathcal{R}_{l_{1}, l_{2}, L, M}^{(1 \mathrm{C})+}\left(r_{1}, r_{2}\right)= & \frac{1}{(2 \pi)^{5 / 2}} \mathcal{T}_{l_{1}, l_{2}, L, M}^{(1 \mathrm{C})}(\kappa \cos \alpha, \kappa \sin \alpha) \frac{\kappa^{3 / 2}}{R^{5 / 2}} \\
& \times \exp \left(i \kappa R+\frac{i \ln \left(2 \kappa R \sin ^{2} \alpha\right)}{\kappa \sin \alpha}+\frac{i \pi}{4}\right),
\end{aligned}
$$

where $\mathcal{T}_{l_{1}, l_{2}, L, M}^{(1 \mathrm{C})}$ are the partial ionization amplitudes in the screening model we are interested in. Instead of the integral (132) we now get, for asymptotically large $R$,

$$
\begin{aligned}
\mathcal{I}_{l_{1}, l_{2}, L, M}^{(1 \mathrm{C})}\left(k_{1}, k_{2} ; R\right)= & \frac{1}{2} R^{5} \int_{0}^{\pi / 2} d \alpha \sin ^{2} \alpha \cos ^{2} \alpha \\
& \times\left(\mathcal{R}_{l_{1}, l_{2}}^{(2 \mathrm{C})-*} \frac{\partial \mathcal{R}_{l_{1}, l_{2}, L, M}^{(1 \mathrm{C})+}}{\partial R}\right. \\
& \left.-\mathcal{R}_{l_{1}, l_{2}, L, M}^{(1 \mathrm{C})+} \frac{\partial \mathcal{R}_{l_{1}, l_{2}}^{(2 \mathrm{C}) *}}{\partial R}\right) .
\end{aligned}
$$

Evaluating this integral following the procedure used in Sec. VI we have

$$
\mathcal{T}_{l_{1}, l_{2}, L, M}^{(\mathrm{C})}\left(k_{1}, k_{2}\right)=\lim _{R \rightarrow \infty} \mathcal{I}_{l_{1}, l_{2}, L, M}^{(\mathrm{C})}\left(k_{1}, k_{2} ; R\right) \exp \left[\frac{i}{k_{1}} \ln \left(\frac{2 k_{1}^{2} R}{\kappa}\right)\right] \text {. }
$$

The first partial wave of the screening model corresponds to the Temkin-Poet model: $\mathcal{T}_{0,0,0,0}^{(1 \mathrm{C})}\left(k_{1}, k_{2}\right) \equiv \mathcal{T}^{(\mathrm{TP})}\left(k_{1}, k_{2}\right)$. The exact Temkin-Poet model for ionization has been numerically solved in [7]. The agreement between the corresponding benchmark ionization amplitude and $\mathcal{T}_{0,0,0,0}^{(1 \mathrm{C})}\left(k_{1}, k_{2}\right)$ from Eq. (146) has been communicated recently [50] (see below for more discussion).

Finally, from the $a c$ form of the ionization amplitude given by Eq. (81) we get the direct integral form of the partial ionization amplitudes

$$
\begin{aligned}
\mathcal{T}_{l_{1}, l_{2}, L, M}^{(1 \mathrm{C})}\left(k_{1}, k_{2}\right)= & \frac{1}{2} \lim _{R \rightarrow \infty} R^{5} \int_{0}^{\pi / 2} d \alpha \sin ^{2} \alpha \cos ^{2} \alpha \\
& \times\left(\mathcal{R}_{l_{1}, l_{2}}^{(1 \mathrm{C}) * *} \frac{\partial \mathcal{R}_{l_{1}, l_{2}, L, M}^{(1 \mathrm{C})+}}{\partial R}\right. \\
& \left.-\mathcal{R}_{l_{1}, l_{2}, L, M}^{(1 \mathrm{C})+} \frac{\partial \mathcal{R}_{l_{1}, l_{2}}^{(1 \mathrm{C}) *}}{\partial R}\right) .
\end{aligned}
$$

We recommend it for practical calculations of the screening model.

\section{B. Model with no electron-electron interaction}

Consider a model electron-hydrogen ionization problem where there is no electron-electron interaction; we call it a $2 \mathrm{C}$ model. The asymptotic form of the unscattered wave function $\Psi^{(f)-}$ for this model is given by Eq. (9) with $z_{1}$ $=z_{2}=1$. The partial waves of this function have also been given earlier in Eq. (130). Using Eqs. (9) (with $z_{1}=z_{2}=1$ ) and (117) we get for the asymptotic form of the corresponding continuum scattered wave

$$
\begin{aligned}
\Phi_{c}^{(2 \mathrm{C})+}\left(\boldsymbol{r}_{1}, \boldsymbol{r}_{2}\right)= & \frac{1}{(2 \pi)^{5 / 2}} T^{(2 \mathrm{C})}\left(\kappa \cos \alpha \hat{\boldsymbol{r}}_{1}, \kappa \sin \alpha \hat{\boldsymbol{r}}_{2}\right) \frac{\kappa^{3 / 2}}{R^{5 / 2}} \\
& \times \exp \left(i \kappa R+\frac{i \ln \left(2 \kappa R \sin ^{2} \alpha\right)}{\kappa \sin \alpha}\right. \\
& \left.+\frac{i \ln \left(2 \kappa R \cos ^{2} \alpha\right)}{\kappa \cos \alpha}+\frac{i \pi}{4}\right)
\end{aligned}
$$

where $T^{(2 \mathrm{C})}$ is the ionization amplitude in this model. This allows us to write the asymptotic form of the partial waves of the scattered wave function for our model:

$$
\begin{aligned}
\mathcal{R}_{l_{1}, l_{2}, L, M}^{(2 \mathrm{C})+}\left(r_{1}, r_{2}\right)= & \frac{1}{(2 \pi)^{5 / 2}} \mathcal{T}_{l_{1}, l_{2}, L, M}^{2 \mathrm{C})}(\kappa \cos \alpha, \kappa \sin \alpha) \frac{\kappa^{3 / 2}}{R^{5 / 2}} \\
& \times \exp \left(i \kappa R+\frac{i \ln \left(2 \kappa R \sin ^{2} \alpha\right)}{\kappa \sin \alpha}\right. \\
& \left.+\frac{i \ln \left(2 \kappa R \cos ^{2} \alpha\right)}{\kappa \cos \alpha}+\frac{i \pi}{4}\right)
\end{aligned}
$$

where $\mathcal{T}_{l_{1}, l_{2}, L, M}^{(2 \mathrm{C})}$ are the corresponding partial ionization amplitudes. Now the integral (132) reads as, for large $R$,

$$
\begin{aligned}
\mathcal{I}_{l_{1}, l_{2}, L, M}^{(2 \mathrm{C})}\left(k_{1}, k_{2} ; R\right)= & \frac{1}{2} R^{5} \int_{0}^{\pi / 2} d \alpha \sin ^{2} \alpha \cos ^{2} \alpha \\
& \times\left(\mathcal{R}_{l_{1}, l_{2}}^{(2 \mathrm{C})-*} \frac{\partial \mathcal{R}_{l_{1}, l_{2}, L, M}^{(2 \mathrm{C})+}}{\partial R}\right. \\
& \left.-\mathcal{R}_{l_{1}, l_{2}, L, M}^{(2 \mathrm{C})+} \frac{\partial \mathcal{R}_{l_{1}, l_{2}}^{(2 \mathrm{C}) *}}{\partial R}\right) .
\end{aligned}
$$

Calculating this integral, after some algebra, we get an integral representation for the ionization amplitude for the model with no electron-electron interaction

$$
\mathcal{T}_{l_{1}, l_{2}, L, M}^{(2 \mathrm{C})}\left(k_{1}, k_{2}\right)=\lim _{R \rightarrow \infty} \mathcal{I}_{l_{1}, l_{2}, L, M}^{(2 \mathrm{C})}\left(k_{1}, k_{2} ; R\right) .
$$

In other words, for this model the partial-wave Peterkop integral and the integral form of the partial ionization amplitudes, which we would get from Eq. (81), coincide. The Peterkop approach to extracting the amplitude is therefore best suited for this model.

\section{Collinear model}

Finally, we consider a model electron-hydrogen ionization problem where all three particles are in line, the so-called collinear (CL) model, relevant to the threshold region. The asymptotic form of the unscattered wave $\Psi^{(f)-}$ for this model is given by

$$
\begin{aligned}
\Psi_{\boldsymbol{k}_{1}, \boldsymbol{k}_{2}}^{(\mathrm{CL})-}\left(\boldsymbol{r}_{1}, \boldsymbol{r}_{2}\right)= & e^{i \boldsymbol{k}_{1} \cdot \boldsymbol{r}_{1}-i \boldsymbol{k}_{2} \cdot \hat{\boldsymbol{r}}_{1} r_{2}} \psi_{1}\left(\boldsymbol{k}_{1}, \boldsymbol{r}_{1}\right) \psi_{1}\left(\boldsymbol{k}_{2},-r_{2} \hat{\boldsymbol{r}}_{1}\right) \\
& \times \psi_{-1 / 2}\left[k_{3},\left(r_{1}+r_{2}\right) \hat{\boldsymbol{r}}_{1}\right] .
\end{aligned}
$$

Then using Eqs. (152) and (117) we get for the scattered wave 


$$
\begin{aligned}
\Phi_{c}^{(\mathrm{CL})+}\left(\boldsymbol{r}_{1}, \boldsymbol{r}_{2}\right)= & \frac{1}{(2 \pi)^{5 / 2}} T^{(\mathrm{CL})}\left(\kappa \cos \alpha \hat{\boldsymbol{r}}_{1},-\kappa \sin \alpha \hat{\boldsymbol{r}}_{1}\right) \frac{\kappa^{3 / 2}}{R^{5 / 2}} \\
& \times \exp \left(i \kappa R+\frac{i \ln \left(2 \kappa R \sin ^{2} \alpha\right)}{\kappa \sin \alpha}\right. \\
& +\frac{i \ln \left(2 \kappa R \cos ^{2} \alpha\right)}{\kappa \cos \alpha}-\frac{i \ln [\kappa R(\cos \alpha+\sin \alpha)]}{\kappa(\cos \alpha+\sin \alpha)} \\
& \left.+\frac{i \pi}{4}\right),
\end{aligned}
$$

where $T^{(\mathrm{CL})}$ is the ionization amplitude corresponding to this model. This allows us to write the asymptotic form of the partial waves of the scattered wave function for the collinear model:

$$
\begin{aligned}
\mathcal{R}_{l_{1}, l_{2}, L, M}^{(\mathrm{CL})+}\left(r_{1}, r_{2}\right)= & \frac{1}{(2 \pi)^{5 / 2}} \mathcal{T}_{l_{1}, l_{2}, L, M}^{\mathrm{CL})}(\kappa \cos \alpha, \kappa \sin \alpha) \frac{\kappa^{3 / 2}}{R^{5 / 2}} \\
& \times \exp \left(i \kappa R+\frac{i \ln \left(2 \kappa R \sin ^{2} \alpha\right)}{\kappa \sin \alpha}\right. \\
& +\frac{i \ln \left(2 \kappa R \cos ^{2} \alpha\right)}{\kappa \cos \alpha} \\
& \left.-\frac{i \ln [\kappa R(\cos \alpha+\sin \alpha)]}{\kappa(\cos \alpha+\sin \alpha)}+\frac{i \pi}{4}\right),
\end{aligned}
$$

where $\mathcal{T}_{l_{1}, l_{2}, L, M}^{(\mathrm{CL})}$ are the partial ionization amplitudes in the CL model. Now for large $R$ the integral (132) takes the form

$$
\begin{aligned}
\mathcal{I}_{l_{1}, l_{2}, L, M}^{(\mathrm{CL})}\left(k_{1}, k_{2} ; R\right)= & \frac{1}{2} R^{5} \int_{0}^{\pi / 2} d \alpha \sin ^{2} \alpha \cos ^{2} \alpha \\
& \times\left(\mathcal{R}_{l_{1}, l_{2}}^{(2 \mathrm{C})-*} \frac{\partial \mathcal{R}_{l_{1}, l_{2}, L, M}^{(\mathrm{CL})+}}{\partial R}\right. \\
& \left.-\mathcal{R}_{l_{1}, l_{2}, L, M}^{(\mathrm{CL})+} \frac{\partial \mathcal{R}_{l_{1}, l_{2}}^{(2 \mathrm{C})-*}}{\partial R}\right) .
\end{aligned}
$$

Calculating this integral, after some algebra, we get an integral representation for the ionization amplitude for the collinear model:

$$
\begin{aligned}
\mathcal{T}_{l_{1}, l_{2}, L, M}^{(\mathrm{CL})}\left(k_{1}, k_{2}\right)= & \lim _{R \rightarrow \infty} \mathcal{I}_{l_{1}, l_{2}, L, M}^{(\mathrm{CL})}\left(k_{1}, k_{2} ; R\right) \\
& \times \exp \left(\frac{i \ln \left[\left(k_{1}+k_{2}\right) R\right]}{\left(k_{1}+k_{2}\right)}\right) .
\end{aligned}
$$

The amplitude magnitudes, via the cross sections, and the phases corresponding to Eqs. (146) and (156) (after taking into account the indistinguishability of the electrons) have been published recently [50] for the Temkin-Poet and socalled collinear $S$-wave models. They are in agreement with similar calculations carried out in [51], where integral representations for Temkin-Poet and collinear $S$-wave models have been obtained. We point out, however, that our $S$-wave amplitudes obtained from Eqs. (146) and (156) are slightly different from those used in [51]. This is because of the ambiguity of the Peterkop wave function used in [51]. As a result in [51] one has to further add some hyperradius- dependent phase [namely, $Q\left(k_{>}, 1\right)$ of [51]] to reproduce the phase obtained in the exact numerical integration [7] and other calculations [50] of the Temkin-Poet model. Apart from this, the agreement between our ionization amplitudes in the Temkin-Poet and collinear $S$-wave models and results of [51] indicates that the simple and transparent approach to calculating the partial waves and amplitudes presented in this work leads to the correct answer.

It may be worthwhile to point out that Eqs. (146) and (156) do not display the factor $\exp \left[i \sigma_{0}\left(k_{1}\right)+i \sigma_{0}\left(k_{2}\right)\right]$ (for comparison, when all angular momenta are set to zero) as opposed to the results of [51]. The reason for this is that in the present work the partial-wave Peterkop integral $\mathcal{I}_{l_{1}, l_{2}, L, M}$ has already a factor $\exp \left[-i \pi\left(l_{1}+l_{2}\right) / 2+i \sigma_{l_{1}}\left(k_{1}\right)+i \sigma_{l_{2}}\left(k_{2}\right)\right]$, which was dropped in [51] [see Eqs. (132) and (130)].

\section{DISCUSSION}

In this section we make a few important remarks based on the results presented in this work. First, as is the case in two-particle scattering, the knowledge of the asymptotic wave function is key to calculating the amplitudes of various processes taking place in a three-body system. For breakup, it is particularly important to clearly understand the differences in the continuum parts of the asymptotic forms of the total scattering wave functions $\Phi_{i}^{+}$and $\Psi_{f}^{-}$. In this paper we denoted them as $\Phi_{c}^{+}$and $\Psi^{(f)-}$, respectively. Often in the literature $\Phi_{c}^{+}$and $\Psi^{(f)-}$ are referred to as two versions (the plane wave and spherical wave) of "one asymptotic wave function" as if they were equivalent or represented the same function. Peterkop [44] even says that asymptotically they should coincide. This is a misunderstanding. The function $\Psi^{(f)-}$ represents the initial unscattered state of the three "free" Coulomb particles [the left-hand side of Eq. (2)], i.e., the Coulomb-modified three-body plane wave. Apart from the modification of the plane wave due to the long-range Coulomb interaction between the three pairs, there is no scattering information in this wave function. It is a state from which the total scattering wave function $\Psi_{f}^{-}$starts to develop [see Eq. (2)]; on the other hand, $\Phi_{c}^{+}$is the continuum part of $\Phi_{i}^{+}$, the wave function which starts from the two-fragment channel [see Eq. (8)]. The wave function $\Phi_{c}^{+}$is formed when the scattering takes place and describes the breakup event. Therefore, by definition, it should carry information about the breakup of an initial bound state of hydrogen which took place and has the form of the outgoing spherical scattered wave. From Eqs. (51)-(54) we see that $\Psi_{f}^{-}$also has a spherical scattered part (containing information about the $3 \rightarrow 3$ process) of the same order as $\Phi_{c}^{+}$; however, it is suppressed by the stronger continuum term $\Psi^{(f)-}$. In other words, $\Psi^{(f)-}$ and $\Phi_{c}^{+}$are completely different functions. Any comparison between the (three-body) plane wave and spherical scattered wave carrying away the information about what happened during the collision is not appropriate. In this sense the idea of using two distinct notations for two different forms of the total wave function suggested in [16] and extended to this work is helpful.

Second, the asymptotic form of the total scattering wave $\Phi_{i}^{+}$in the domain where all interparticle distances are large 
$\left(\Omega_{0}\right)$ is sometimes called semiclassical. In this regard we emphasize that the form obtained in [17] for all possible asymptotic regions $\Omega_{i}, i=0-3$ [see Eqs. (19)-(22)], is fully quantum mechanical. In $\Omega_{0}$ the semiclassical approach does give the same result as the quantum-mechanical one. However, the semiclassical approach is not applicable in all asymptotic domains other than $\Omega_{0}$. We also note that the similar asymptotic form of the total scattering wave $\Psi_{f}^{-}$presented in this work [see Eqs. (51)-(54)] is also fully quantum mechanical.

Third, from Eq. (127) we could write

$$
\begin{aligned}
\mathcal{T}_{l_{1}, l_{2}, L, M}\left(k_{1}, k_{2}\right)= & \lim _{R \rightarrow \infty} \frac{(2 \pi R)^{5 / 2}}{\kappa^{3 / 2}} \frac{\mathcal{R}_{l_{1}, l_{2}, L, M}^{+}\left(r_{1}, r_{2}\right)}{\tau_{l_{1}, l_{2}, L}\left(k_{1}, k_{2} ; R k_{1} / \kappa, R k_{2} / \kappa\right)} \\
& \times \exp \left(-i \kappa R-\frac{i}{k_{1}} \ln \left(2 R k_{1}^{2} / \kappa\right)\right. \\
& \left.-\frac{i}{k_{2}} \ln \left(2 R k_{2}^{2} / \kappa\right)-\frac{i \pi}{4}\right) .
\end{aligned}
$$

Then, taking into account Eq. (139) we get

$$
\left|\mathcal{T}_{l_{1}, l_{2}, L, M}\left(k_{1}, k_{2}\right)\right|=\lim _{R \rightarrow \infty} \frac{(2 \pi R)^{5 / 2}}{\kappa^{3 / 2}}\left|\mathcal{R}_{l_{1}, l_{2}, L, M}^{+}\left(r_{1}, r_{2}\right)\right| .
$$

Similarly, for often used models we have

$$
\begin{aligned}
\mathcal{T}_{l_{1}, l_{2}, L, M}^{(\mathrm{C})}\left(k_{1}, k_{2}\right)= & \lim _{R \rightarrow \infty} \frac{(2 \pi R)^{5 / 2}}{\kappa^{3 / 2}} \mathcal{R}_{l_{1}, l_{2}, L, M}^{(1 \mathrm{C})+}\left(r_{1}, r_{2}\right) \\
& \times \exp \left(-i \kappa R-\frac{i}{k_{2}} \ln \left(2 R k_{2}^{2} / \kappa\right)-\frac{i \pi}{4}\right), \\
\mathcal{T}_{l_{1}, l_{2}, L, M}^{\mathrm{CL})}\left(k_{1}, k_{2}\right)= & \lim _{R \rightarrow \infty} \frac{(2 \pi R)^{5 / 2}}{\kappa^{3 / 2}} \mathcal{R}_{l_{1}, l_{2}, L, M}^{(\mathrm{CL})+}\left(r_{1}, r_{2}\right) \\
& \times \exp \left(-i \kappa R-\frac{i}{k_{1}} \ln \left(2 R k_{1}^{2} / \kappa\right)\right. \\
& -\frac{i}{k_{2}} \ln \left(2 R k_{2}^{2} / \kappa\right)+\frac{i}{k_{1}+k_{2}} \\
& \left.\times \ln \left[2 R\left(k_{1}^{2}+k_{2}^{2}\right) / \kappa\right]-\frac{i \pi}{4}\right) .
\end{aligned}
$$

The wave functions $\mathcal{R}_{l_{1}, l_{2}, L, M}^{+}, \mathcal{R}_{l_{1}, l_{2}, L, M}^{(1 \mathrm{C})+}$, and $\mathcal{R}_{l_{1}, l_{2}, L, M}^{(\mathrm{CL})+}$ in these equations all come from the solution of the corresponding Schrödinger equations. In principle, these relationships [and relationships similar to Eq. (158) for $\left|\mathcal{T}_{l_{1}, l_{2}, L, M}^{(1 \mathrm{C})}\right|$ and $\left.\left|\mathcal{T}_{l_{1}, l_{2}, L, M}^{(\mathrm{CL})}\right|\right]$ can be used to obtain the corresponding partial ionization amplitudes or their magnitudes. Due to their simplicity they may even prove to be an easier choice than the integral forms. However, this procedure is prone to certain errors associated with the premature use of the stationaryphase relations (134) and numerically sensitive. Nevertheless, they can be used for simple estimates.
Finally, having calculated the asymptotic form of the scattered wave in partial waves we have got a clue to an original method of solving the Schrödinger equation for ionization. Usually, this equation is solved for the total scattering wave function $\Phi_{i}^{+}$or its scattered part $\Phi_{i}^{(\mathrm{sc})+}$, which is increasingly oscillatory with increasing radius and has a divergent phase. If one uses our results, Eq. (127) for the full problem and Eqs. (144) and (154) for the Temkin-Poet and collinear models, in the original Schrödinger equation for the radial wave function, then one gets (as a result of the variable transformation) a new equation for the corresponding ionization amplitude. As we extend the radius of integration of the new differential equation, its solution reaches a constant which is in fact the ionization amplitude of interest. We suppose that it is easier to propagate to large distances a constant solution rather than an oscillatory one.

\section{CONCLUSION}

A general formulation of the theory of ionization of atoms by electron impact has been given. A divergence-free representation for the ionization amplitude of atomic hydrogen by electron impact has been presented. In the formulation, the ionization amplitude takes four alternative surface-integral forms ideal for practical calculations. The formulation has also been extended to amplitudes for all possible reactions taking place in the scattering system. This was done in a general way for arbitrary three-body systems. The present formulation was then shown to lead to a well-defined post form of the breakup amplitude valid for arbitrary potentials including the long-range Coulomb interaction.

Furthermore, another fundamental problem has been addressed. The partial-wave expansion for the asymptotic forms of the total scattering wave function, developed from both the initial and the final states, was derived. These expansions are necessary for calculating electron-impact ionization in methods based on direct integration of the Schrödinger equation on a two-dimensional radial lattice. The integral representation was then extended to partial ionization amplitudes. A rigorous proof was given of the relationship used in ECS-based calculations of the cross sections for the full electron-hydrogen ionization problem.

The utility of the presented analysis was demonstrated using two well-known model problems. Exact asymptotic forms of the scattered wave functions have been given.

The formalism presented is readily applicable to extraction of the exact amplitudes in direct calculations of other atomic and molecular breakup processes including the double photoionization of helium or breakup and photodisintegration calculations in nuclear physics. It may also be useful in further developing the effective-charge perturbation approaches. The partial-wave forms of the three-body wave functions presented are capable of reducing the sixdimensional integrals used in the distorted-wave Born approximations to two-dimensional ones.

\section{ACKNOWLEDGMENTS}

The work was supported by the Australian Research Council, U.S. DOE Grant No. DE-FG03-93ER40773, and NSF Grant No. PHY-0140343. 
[1] A. Temkin, Phys. Rev. 126, 130 (1962).

[2] R. Poet, J. Phys. B 13, 2995 (1980).

[3] J. Botero and J. Shertzer, Phys. Rev. A 46, R1155 (1992).

[4] Y. D. Wang and J. Callaway, Phys. Rev. A 48, 2058 (1993).

[5] J. Shertzer and J. Botero, Phys. Rev. A 49, 3673 (1994).

[6] Y. D. Wang and J. Callaway, Phys. Rev. A 50, 2327 (1994).

[7] S. Jones and A. T. Stelbovics, Phys. Rev. Lett. 84, 1878 (2000).

[8] M. Baertschy, T. N. Rescigno, and C. W. McCurdy, Phys. Rev. A 64, 022709 (2001).

[9] T. N. Rescigno, M. Baertschy, W. A. Isaacs, and C. W. McCurdy, Science 286, 2474 (1999).

[10] P. L. Bartlett, A. T. Stelbovics, and I. Bray, J. Phys. B 37, L69 (2004).

[11] I. Bray, D. V. Fursa, A. S. Kheifets, and A. T. Stelbovics, J. Phys. B 35, R117 (2002).

[12] K. Bartschat, E. T. Hudson, M. P. Scott, P. G. Burke, and V. M. Burke, J. Phys. B 29, 115 (1996).

[13] M. S. Pindzola, D. Mitnik, and F. Robicheaux, Phys. Rev. A 62, 062718 (2000).

[14] A. S. Kadyrov, A. M. Mukhamedzhanov, A. T. Stelbovics, and I. Bray, Phys. Rev. Lett. 91, 253202 (2003).

[15] R. K. Peterkop, Izv. Akad. Nauk Latv. SSR Riga 9, 79 (1960).

[16] A. S. Kadyrov, A. M. Mukhamedzhanov, and A. T. Stelbovics, Phys. Rev. A 67, 024702 (2003).

[17] A. S. Kadyrov, A. M. Mukhamedzhanov, A. T. Stelbovics, I. Bray, and F. Pirlepesov, Phys. Rev. A 68, 022703 (2003).

[18] C. R. Garibotti and J. E. Miraglia, Phys. Rev. A 21, 572 (1980).

[19] M. Brauner, J. S. Briggs, and H. Klar, J. Phys. B 22, 2265 (1989).

[20] L. Malegat, P. Selles, and A. Kazansky, Phys. Rev. Lett. 85, 4450 (2000).

[21] C. Pan and A. F. Starace, Phys. Rev. Lett. 67, 185 (1991).

[22] J. Berakdar and J. S. Briggs, Phys. Rev. Lett. 72, 3799 (1994).

[23] M. Pont, R. Shakeshaft, F. Maulbetsch, and J. S. Briggs, Phys. Rev. A 53, 3671 (1996).

[24] S. Jones, D. H. Madison, A. Franz, and P. L. Altick, Phys. Rev. A 48, R22 (1993).

[25] S. P. Lucey, J. Rasch, and C. T. Whelan, Proc. R. Soc. London, Ser. A 455, 349 (1999).

[26] R. G. Newton, Scattering Theory of Waves and Particles, 2nd ed., Texts and Monographs in Physics (Springer, New York, 1982).

[27] S. Jones, D. H. Madison, and M. Baertschy, Phys. Rev. A 67, 012703 (2003).

[28] R. K. Peterkop, Opt. Spektrosk. 13, 153 (1962) [Opt. Spec- trosc. 13, 87 (1962)].

[29] R. K. Peterkop, Theory of Ionization of Atoms by Electron Impact (Colorado Associated University Press, Boulder, CO, 1977).

[30] M. R. H. Rudge, Rev. Mod. Phys. 40, 564 (1968).

[31] The wave functions $\Psi_{f}^{-}$and $\Phi_{i}^{+}$are in fact two forms of the total scattering wave function of the three-body system, having two different starting points.

[32] G. Arfken, Mathematical Methods for Physicists, 2nd ed. (Academic, New York, 1971).

[33] C. W. McCurdy, D. A. Horner, and T. N. Rescigno, Phys. Rev. A 63, 022711 (2001).

[34] N. Bleistein and R. A. Handelsman, Asymptotic Expansions of Integrals (Dover, New York, 1986).

[35] Strictly speaking, the amplitude of ionization of state $i$ should be denoted as $T\left(\boldsymbol{k}_{1}, \boldsymbol{k}_{2} ; \boldsymbol{k}_{i}\right)$ which shows the momentum of the incident electron explicitly. However, for brevity we use the shorter notation $T\left(\boldsymbol{k}_{1}, \boldsymbol{k}_{2}\right)$. For the recombination amplitudes, a shorter notation is not appropriate.

[36] E. O. Alt and A. M. Mukhamedzhanov, Phys. Rev. A 47, 2004 (1993).

[37] A. M. Mukhamedzhanov and M. Lieber, Phys. Rev. A 54, 3078 (1996).

[38] D. A. Varshalovich, A. N. Moskalev, and V. K. Khersonskii, Quantum Theory of Angular Momentum, 1st ed. (World Scientific, Philadelphia, 1988).

[39] R. Peterkop and L. Rabik, J. Phys. B 5, 1823 (1972).

[40] P. L. Altick, Phys. Rev. A 21, 1381 (1980).

[41] P. L. Altick, Phys. Rev. A 25, 128 (1982).

[42] P. L. Altick, J. Phys. B 16, 3543 (1983).

[43] R. Peterkop and A. Liepinsh, J. Phys. B 14, 2763 (1981).

[44] R. Peterkop, J. Phys. B 15, L751 (1982).

[45] R. Peterkop and A. Liepinsh, J. Phys. B 18, 2763 (1985).

[46] M. Gailitis and R. Peterkop, J. Phys. B 22, 1231 (1989).

[47] S. P. Merkuriev and L. D. Faddeev, Kvantovaja Teoria Rasseijania dlja Sistem Neskolkih Chastits (Nauka, Moscow, 1985) [L. D. Faddeev and S. P. Merkuriev, Quantum Scattering Theory for Several Particle Systems (Kluwer, Dordrecht, 1993)].

[48] M. Gailitis, Dokl. Akad. Nauk SSSR 312, 354 (1990).

[49] M. Gailitis, J. Phys. B 23, 85 (1990).

[50] P. L. Bartlett, I. Bray, S. Jones, A. T. Stelbovics, A. S. Kadyrov, K. Bartschat, G. ver Steeg, M. P. Scott, and P. G. Burke, Phys. Rev. A 68, 020702(R) (2003).

[51] T. N. Rescigno, M. Baertschy, and C. W. McCurdy, Phys. Rev. A 68, 020701(R) (2003). 